\title{
Near-IR period-luminosity relations for pulsating stars in $\omega$ Centauri (NGC 5139) ${ }^{\star}$
}

\author{
C. Navarrete ${ }^{1,2}$, M. Catelan ${ }^{1,2,3}$, R. Contreras Ramos ${ }^{2,1}$, J. Alonso-García ${ }^{4,2,1}$, \\ F. Gran ${ }^{1,2}$, I. Dékány ${ }^{5}$, and D. Minniti $6,2,7$ \\ ${ }^{1}$ Instituto de Astrofísica, Pontificia Universidad Católica de Chile, Av. Vicuña Mackenna 4860, 782-0436 Macul, Santiago, Chile \\ e-mail: [cnavarre;mcatelan;rcontre]@astro.puc.cl \\ 2 Instituto Milenio de Astrofísica, Santiago, Chile \\ ${ }^{3}$ Centro de Astro-Ingeniería, Pontificia Universidad Católica de Chile, Santiago, Chile \\ ${ }^{4}$ Unidad de Astronomía, Facultad Cs. Básicas, Universidad de Antofagasta, Av. U. de Antofagasta 02800, Antofagasta, Chile \\ 5 Astronomisches Rechen-Institut, Zentrum für Astronomie der Universität Heidelberg, Mönchhofstr. 12-14, 69120 Heidelberg, \\ Germany \\ ${ }^{6}$ Departamento de Fisica, Facultad de Ciencias Exactas, Universidad Andres Bello, Av. Fernandez Concha 700, Las Condes, \\ 4860 Santiago, Chile \\ 7 Vatican Observatory, V00120 Vatican City State, Italy
}

Received 21 November 2016 / Accepted 7 April 2017

\begin{abstract}
Aims. The globular cluster $\omega$ Centauri (NGC 5139) hosts hundreds of pulsating variable stars of different types, thus representing a treasure trove for studies of their corresponding period-luminosity (PL) relations. Our goal in this study is to obtain the PL relations for RR Lyrae and SX Phoenicis stars in the field of the cluster, based on high-quality, well-sampled light curves in the near-infrared (IR).

Methods. Observations were carried out using the VISTA InfraRed CAMera (VIRCAM) mounted on the Visible and Infrared Survey Telescope for Astronomy (VISTA). A total of 42 epochs in $J$ and 100 epochs in $K_{\text {S }}$ were obtained, spanning 352 days. Point-spread function photometry was performed using DoPhot and DAOPHOT crowded-field photometry packages in the outer and inner regions of the cluster, respectively.

Results. Based on the comprehensive catalog of near-IR light curves thus secured, PL relations were obtained for the different types of pulsators in the cluster, both in the $J$ and $K_{\mathrm{S}}$ bands. This includes the first PL relations in the near-IR for fundamental-mode SX Phoenicis stars. The near-IR magnitudes and periods of Type II Cepheids and RR Lyrae stars were used to derive an updated true distance modulus to the cluster, with a resulting value of $(m-M)_{0}=13.708 \pm 0.035 \pm 0.10 \mathrm{mag}$, where the error bars correspond to the adopted statistical and systematic errors, respectively. Adding the errors in quadrature, this is equivalent to a heliocentric distance of $5.52 \pm 0.27 \mathrm{kpc}$.
\end{abstract}

Key words. stars: variables: RR Lyrae - stars: variables: Cepheids - stars: variables: delta Scuti - infrared: stars - surveys globular clusters: individual: NGC 5139

\section{Introduction}

The globular cluster $\omega$ Centauri (NGC 5139, C1323-472) is the most luminous, massive, and biggest globular cluster (GC) in the Milky Way. Located at RA $=13: 26: 47.28$ and Dec $=-47: 28: 46.1$ (J2000), it appears visible with the naked eye from the southern celestial hemisphere. It has been extensively studied, since it is bright $\left(M_{V} \sim-10.26 \mathrm{mag}\right)$, large (apparent size of $36^{\prime}$ ), and nearby, at a distance of only $5.2 \mathrm{kpc}$, according to the 2010 version of the Harris $(1996,2010)$ catalog of GC parameters. Additionally, it hosts millions of stars in a field covering $\sim 1.5 \times 1.5 \mathrm{deg}^{2}$, which has led to numerous works devoted to its internal dynamics (van de Ven etal. 2006; van der Marel \& Anderson 2010; D'Souza \& Rix 2013), a suggested extragalactic origin, and its possible

$\star$ Based on observations collected at the European Organisation for Astronomical Research in the Southern Hemisphere, Chile, with the VISTA telescope (project ID 087.D-0472, PI R. Angeloni). associated tidal debris (e.g., Meylan et al. 2001; Dinescu 2002; Altmann et al. 2005; Meza et al. 2005; Bekki \& Norris 2006; Da Costa \& Coleman 2008; Valcarce \& Catelan 2011; Fernández-Trincado et al. 2015; Navarrete et al. 2015a). Naturally, it has also been the subject of extensive variability studies, which is also the main subject of the present work.

In this context, $\omega$ Cen stands out as one of the three most RR Lyrae (RRL)-rich GCs known (Navarrete et al. 2015b). Moreover, it is the GC with the highest known number of SX Phe stars (74; Olech et al. 2005; Weldrake et al. 2007; Cohen \& Sarajedini 2012). At the same time, while the number of type II Cepheids (T2Cs) that it is known to harbor (seven) may seem small in an absolute sense, $\omega$ Cen still holds the record as the most T2C-rich of any GC (Clement et al. 2001; Matsunaga et al. 2006). Also, even though anomalous Cepheids (ACEPs) are primarily found in nearby extragalactic environments (Catelan \& Smith 2015), an ACEP classification has been 
advanced for some of the RRL stars in $\omega$ Cen (Nemec et al. 1994). The existence of ACEPs in $\omega$ Cen would thus be another indication of its possible extragalactic origin. However, none of the ACEP candidates has been confirmed or definitely rejected yet, probably because they are quite similar to the longest-period RRc stars in terms of periods and light curve shapes.

At least two stellar populations are known in $\omega$ Cen, with metallicity peaks centered at $[\mathrm{Fe} / \mathrm{H}] \approx-1.6$ and $-1.1 \mathrm{dex}$ (Bedin et al. 2004; Joo \& Lee 2013), and additional peaks likely also being present (e.g., Johnson et al. 2009). Besides metallicity, it has been reported that these populations differ in their detailed chemistry and age, with the metal-richer stars likely being enhanced in helium by $\Delta Y \sim 0.17$ (Norris 2004; Piotto et al 2005; Dupree et al. 2011; Dupree \& Avrett 2013; Tailo et al 2016, and references therein). Despite the strong evidence for large He-abundance spread among $\omega$ Cen's stars, Sollima et al. (2006a) did not detect variations in $Y$ in their study of the pulsation properties (and spectroscopic metallicities) of the RRL stars in the cluster. Later, Marconi et al. (2011) studied the impact of the helium content on the RRL properties based on evolutionary and pulsational models, finding that the helium content has a marginal effect on the pulsation properties of these variable stars. Very recently, Tailo et al. (2016) claimed consistency between the predicted and observed properties of RRL stars in $\omega$ Cen but with a relatively small spread in helium, amounting to $\Delta Y \sim 0.03$

In this context, the different, well-represented variability types that are simultaneously present in $\omega$ Cen offer us a unique opportunity to perform a comparison of their properties. In particular, the pulsating stars present in the cluster are wellknown distance indicators, following period-luminosity (PL) relations that are often used to derive distances (Catelan \& Smith 2015, and references therein). We note, in this sense, that PL relations at infrared wavelengths have some advantages compared to the ones derived at visible bands (first highlighted by McGonegal et al. 1983, in a study of the PL relations of Classical Cepheids): the amplitude of the variables is smaller, leading to a smaller error in the derived mean magnitudes, even when relatively few data points are available; the interstellar extinction is lower $\left(\mathcal{A}_{K} / \mathcal{A}_{V} \sim 1 / 10\right)$; and the infrared luminosities are less sensitive to temperature changes, leading to tighter PL relations, compared to the visible regime. Among the pulsating variability types present in $\omega$ Cen, the primary distance indicators are the RRLs, which are not only relatively bright (being horizontal-branch [HB] stars) but also significantly more numerous than the other types of pulsators that are present in the cluster. RRL stars follow a well-defined PL relation at the near-IR, at odds with the visual where such a relation is not present (see, e.g., Catelan \& Smith 2015). The near-IR PL relations were first discovered in the pioneering work by Longmore et al. (1986), based on the study of three globular clusters. A detailed theoretical derivation in the Johnson-Cousin-Glass $U B V R I J H K$ system was performed by Catelan et al. (2004), who showed how the PL relation first appears in the $R$ band, decreasing its scatter towards redder bands, becoming tightest in the $K$ regime. From the observational side, Klein \& Bloom (2014) studied RRLs from the field in 13 different photometric bandpasses, including the midinfrared Wide-Field Infrared Survey Explorer (WISE) bands, confirming that the PL relation does become tighter in the infrared (see also Klein et al. 2014).

SX Phe stars are the faintest among the known pulsating stars in the cluster, and thus more challenging to observe than their brighter siblings - and yet, they are the second most numerous type of variable stars currently known in $\omega$ Cen.
The study of the PL relations for SX Phe and their Population I counterparts, the $\delta$ Scuti stars, is complicated by the need to properly identify the pulsation modes, as these stars are often pulsating in more than one mode simultaneously. In these cases, the dominant mode is often the radial fundamental, first, or second overtone, though non-radial $p$ modes are often also present (Catelan \& Smith 2015, and references therein). The PL relations of these stars have been investigated using theoretical models (e.g., Petersen \& Christensen-Dalsgaard 1999; Templeton et al. 2002) and observational data in the optical (Nemec et al. 1994; McNamara 1995; Alcock et al. 2000; McNamara 2011; Cohen \& Sarajedini 2012) alike, but a clear consensus has not yet been reached regarding, for instance, the impact of the metallicity on these PL relations. An extension of these PL studies to the near-IR regime has not been carried out yet, and in fact is expected to present some important challenges: since many SX Phe stars are small-amplitude pulsators (with amplitudes below 0.2 mag or so in $V$ ), their amplitudes in the near-IR could easily become similar to, or even lower than, the photometric errors at their corresponding magnitudes. Such small amplitudes are detrimental to the proper characterization of the (often multiple) pulsation modes of the star, as needed to cleanly establish their PL relations. On the other hand, the smaller amplitudes make it easier to compute representative average magnitudes and colors from the time-series data.

T2Cs are considerably fewer in number than either RRL or SX Phe, and only seven are known in $\omega$ Cen. Despite their brightness, the T2C PL relation has not been as extensively studied, undoubtedly due to the fact that they are so few in number (see Catelan \& Smith 2015, for a review and extensive references). As in the case of the RRL stars, the PL relations for T2Cs have also been shown to become tighter at longer wavelengths (Matsunaga et al. 2006, and references therein). In the case of ACEPs, which are even fewer in number in GCs (but not in nearby dwarf galaxies, where they can be very numerous), PL relations in different bandpasses, including the near-IR, have recently been provided by Ripepi et al. (2014a).

Our team has performed a systematic monitoring, in the $J$ and $K_{\mathrm{S}}$ bands, of $\omega$ Cen, with the main goal of obtaining a large number of well-defined light curves for the different types of variables present in this cluster. These well-defined light curves can play an important role as templates for the automated classification of variable stars in near-IR surveys (Angeloni et al. 2014), including, in particular, the VISTA Variables in the Vía Láctea (VVV; e.g., Minniti et al. 2010; Catelan et al. 2011; Saito et al. 2012; Catelan et al. 2013a,b) survey and the VISTA survey of the Magellanic Clouds system (VMC; e.g., Cioni et al. 2011; Ripepi et al. 2014a, 2016) both of which are time-resolved European Southern Observatory (ESO) Public Surveys conducted in the near-IR that run on ESO's VISTA telescope.

This paper is the second in a series dedicated to presenting the results of our $\omega$ Cen near-IR variability survey. In the first paper, Navarrete et al. (2015b) studied the RRL population (in terms of variability type, amplitudes, and membership). Here we present the near-IR PL relations for the different types of pulsating variable stars that are present in the cluster. In the next and final paper of the series, Navarrete et al. (in prep.) will present the derived near-IR photometric catalog for all detected variables in $\omega$ Cen, including not only the pulsating stars but also eclipsing binaries, ellipsoidal variables, and others.

This paper is organized as follows. The observations and data reduction are presented in the next section. Section 3 is devoted to the PL relations for the different types of pulsating stars in $\omega$ Cen. The implied pulsational distance modulus of the cluster 
and the sources of uncertainties are discussed in Sect. 4. Conclusions are presented in Sect. 5.

\section{Observations}

The VIRCAM camera (Dalton et al. 2006), mounted on the VISTA telescope (Emerson \& Sutherland 2010), was used to monitor $\omega$ Cen, obtaining 42 and 100 epochs in the $J$ and $K_{\mathrm{S}}$ bands, respectively. The effective field of view (FoV) of VISTA $\left(1.1 \times 1.5 \mathrm{deg}^{2}\right)$ is large enough to encompass all the pulsating stars known in the field of the cluster, except for four RRL located farther away than the cluster's tidal radius and which are thus likely non-members (Navarrete et al. 2015b).

The characteristics of the observations and data reduction are the same as those already explained in Navarrete et al. (2013, 2015b), and will accordingly not be repeated here. Point-spread function (PSF) photometry was performed using the photometry packages DoPhot (Schechter et al. 1993; Alonso-García et al. 2012) for the outer regions, and DAOPHOT II/ALLFRAME (Stetson 1987, 1994) for the central part of the cluster (i.e., the innermost $\sim 10^{\prime}$ ). All the magnitudes are presented in the VISTA photometric system.

\section{Results}

From our time-series photometry, light curves with the clear signatures of variability were derived for 209 pulsating stars, including seven T2Cs, 89 fundamental-mode (ab-type) and 98 first-overtone (c-type) RRL, 12 SX Phe, and three stars of uncertain type. Intensity-averaged magnitudes were obtained for all of these stars, based on Fourier fits to their light curves. For an additional 62 SX Phe and four RRc stars, variability was not reliably recovered on the basis of our data alone, most likely due to these stars have very small amplitudes in the near-IR (they were previously classified as variables by Kaluzny et al. 2004, based on optical photometry). In these cases, the mean magnitudes were obtained directly from the photometric processing of the images, without any attempt to phase-fit the light curves.

In order to compare the magnitudes and periods with wellcalibrated near-IR PL relations, both $J$ and $K_{\mathrm{S}}$ magnitudes were de-reddened, adopting a color excess of $E(B-V)=0.12 \mathrm{mag}$ from the Harris $(1996,2010)$ online catalog, and the ratios $\mathcal{A}_{X} / E(B-V)$ of 0.866 and 0.364 for the VISTA $J$ and $K_{\mathrm{S}}$ magnitudes, respectively (Catelan et al. 2011).

\subsection{Type II Cepheids}

Light curves for all the T2Cs present in the cluster were recovered, both in $J$ and $K_{\mathrm{S}}$. However, the RV Tau star V1 has a magnitude near the saturation limit in the detector where it was observed. In particular, to derive its light curve, aperture photometry measurements (provided by the Cambridge Astronomical Survey Unit [CASU] catalogs) instead of PSF photometry were used because in the latter V1 is considered a saturated star. Because of this, the individual magnitude measurements have photometric errors higher than for the other T2Cs, and the errors adopted for the intensity-averaged magnitude, in $J$ and $K_{\mathrm{S}}$, are twice as high as the error adopted for the intensity-average magnitudes of the other T2Cs. Figure 1 shows the intensityaveraged magnitudes versus $\log P$ for the seven T2Cs in $\omega$ Cen, for the $J$ and $K_{\mathrm{S}}$ bands. Dashed and dotted lines show the empirical PL relation derived by Matsunaga et al. (2006), shifted by 13.62 and $13.708 \mathrm{mag}$, respectively. The former value corresponds to the distance modulus adopted by Matsunaga et al. for

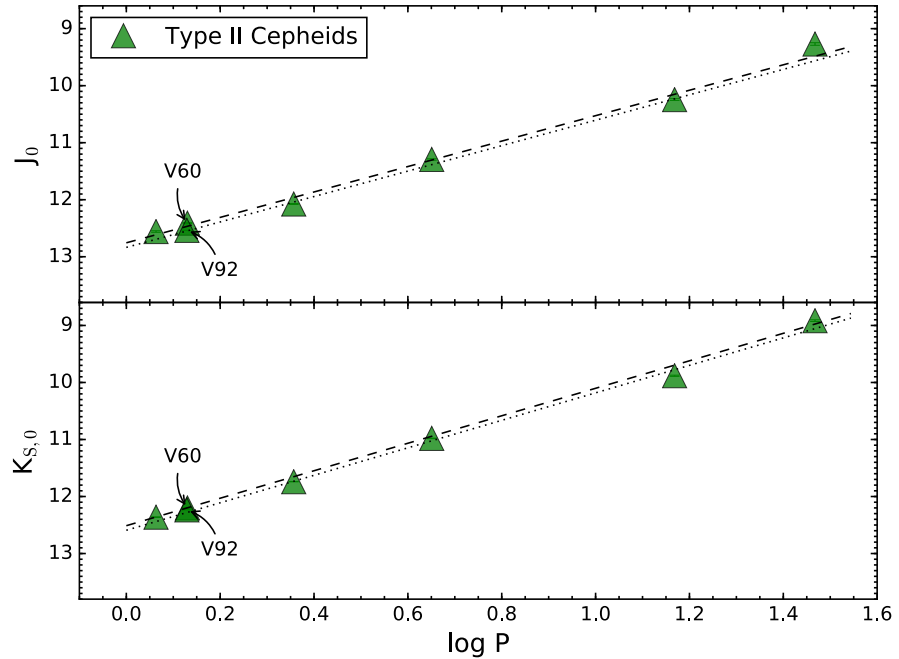

Fig. 1. De-reddened $J$ and $K_{\mathrm{S}}$ magnitudes versus period (in units of days) for the seven known T2Cs in $\omega$ Cen. The dashed lines correspond to the empirical PL relations derived by Matsunaga et al. (2006), shifted to the distance modulus of the cluster used in that study, $13.62 \mathrm{mag}$, while the dotted lines are the same relations, but shifted to our derived distance modulus of 13.708 mag (see Sect. 4). V60 and V92, with roughly the same period and magnitude, are labeled accordingly.

$\omega$ Cen, based on their adopted relation between HB luminosity and metallicity, while the latter one is the final distance modulus obtained in this study (see Sect. 4). As can be noted, all the variables closely follow a well-defined linear relation, irrespective of the distance modulus adopted, showing very little scatter (with a rms in the residuals of the weighted least-squares fit of 0.07 and $0.03 \mathrm{mag}$ in the $J$ and $K_{\mathrm{S}}$ bands, respectively) which is consistent with all of the T2Cs pulsating in the same pulsation mode, and with a negligible dependence of their PL relation on metallicity (and helium). Indeed, Matsunaga et al. (2006) found that all T2Cs in Galactic GCs pulsate in the fundamental mode, and a similar conclusion was reached by Ripepi et al. (2015) in a study of 130 T2Cs in the Large Magellanic Cloud. Matsunaga et al. also found that the metallicity dependence of the PL relation is not very significant, with a zero point dependence of only $(-0.1 \pm 0.06) \mathrm{mag} / \mathrm{dex}$ in the $K_{\mathrm{S}}$ band. Based on theoretical models for BL Her stars (the short-period T2Cs), Di Criscienzo et al. (2007) similarly found a dependence of only $\sim 0.04$ and 0.06 mag dex ${ }^{-1}$ in $J$ and $K_{\mathrm{S}}$, respectively.

While Fig. 1 is consistent with all T2Cs pulsating in the fundamental mode, the previous study of Nemec et al. (1994) claimed that V1, V43, and V60 are actually first-overtone pulsators, while V29, V48, V61, and V92 pulsate in the fundamental mode. However, Fig. 1 does not show parallel sequences occupied by the different stars, as would be expected if they pulsated in different modes (Catelan \& Smith 2015). In fact, the pair represented by V60 and V92 overlap in Fig. 1, the former being brighter than the latter by only 0.15 and 0.05 mag in $J$ and $K_{\mathrm{S}}$, respectively.

Interestingly, V43 and V60 appear consistently brighter than the other stars at the same period (by 0.079 and 0.052 mag in $J$ and $K_{\mathrm{S}}$, respectively) when the PL relation of Matsunaga et al. (2006) is adopted, shifted to the distance modulus derived with the fundamental-mode candidates. We note that V1 deviates more dramatically from the Matsunaga et al. relation, being brighter by $\sim 0.25 \mathrm{mag}$ in $J$ and $\sim 0.12 \mathrm{mag}$ in $K_{\mathrm{S}}-$, but this is likely to be, at least in part, due to saturation (see above). Whether the measured shifts for V43 and V60 would be 
Table 1. $\omega$ Cen weighted-average distance modulus, based on T2Cs.

\begin{tabular}{lccc}
\hline \hline Sub-sample & $N_{\star}$ & $\begin{array}{c}\left(J-M_{J}\right)_{0} \\
(\mathrm{mag})\end{array}$ & $\begin{array}{c}\left(K_{\mathrm{S}}-M_{K_{\mathrm{S}}}\right)_{0} \\
(\mathrm{mag})\end{array}$ \\
\hline T2Cs (all) & 7 & $13.659 \pm 0.121$ & $13.646 \pm 0.066$ \\
T2Cs (all minus V1) & 6 & $13.663 \pm 0.073$ & $13.646 \pm 0.061$ \\
Fundamental-mode candidates only & 4 & $13.686 \pm 0.049$ & $13.664 \pm 0.061$ \\
\hline
\end{tabular}

consistent with the stars pulsating in the first overtone should be further investigated by theoretical modeling of the PL relation for these stars.

Adopting the calibrated PL relation derived by Matsunaga et al. (2006), the distances for the seven T2Cs can be obtained from their intensity-averaged de-reddened $J$ and $K_{\mathrm{S}}$ magnitudes. Table 1 shows the true distance modulus of the cluster, determined using the weighted average of individual measurements in each bandpass, in three cases: (1) using all the T2Cs of the cluster; (2) discarding V1 (the only RV Tau star) due to the aforementioned saturation effects; (3) considering only the fundamental-mode candidates according to Nemec et al. (1994; i.e., V29, V48, V61, and V92). Reassuringly, and as can be seen from the table, the final derived distance modulus does not depend on either the selected sub-sample or the adopted bandpass.

\subsection{RR Lyrae stars}

In order to conduct the most accurate study of the RRL PL relation, we first cleaned the sample, which contains 191 RRLs with measured $J$ and $K_{\mathrm{S}}$ magnitudes, by applying the following cuts. First, the field RRL stars V168, V181, V183, and V283 from Kaluzny et al. (2004), and NV457 and NV458 from Navarrete et al. (2015b) were excluded, since they are not members of the cluster (Navarrete et al. 2015b). Next, V165 and V366 were also dropped, as they have periods of $\sim 0.5$ and 1.0 day, respectively, which does not allow the recovery of the full light curves. Finally, the RRc stars V349 and V351, which are located in the innermost region of the cluster and have inaccurate magnitudes, were discarded as well. As a result, a final sample of $83 \mathrm{RRab}$ and $100 \mathrm{RRc}$ stars was considered in our study of the PL relations in $J$ and $K_{\mathrm{S}}$. Their positions in the corresponding $\log P$-magnitude diagrams are shown in Fig. 2.

The four brightest RRL stars with periods $\sim 0.63$ days $(\log P \sim-0.2)$, namely V84, V118, V135, and V139, have been referred to as "overluminous" previously by Del Principe et al. (2006), but from the examination of the Hubble Space Telescope (HST) catalog of Anderson \& van der Marel (2010), all but V84 have very near companions which could lead to unresolved blends (see Navarrete et al. 2015b). V52 and V143 were not included in the study of Del Principe et al. (2006) but were found to be brighter than other RRab stars because of marked blends (Navarrete et al. 2015b). From this point onwards, these five RRab stars will be discarded from our analysis. V84 is an almost isolated star and its brightness appears to be intrinsic, which could be because it is a foreground RRL or, as Nemec et al. (1994) commented, an ACEP candidate. This possibility will be addressed in Sect. 3.3 and, for now, the star will also be discarded from the RRL sample.

In Fig. 2 there are three RRc stars that appear a little above the other RRc stars with the same period. They are V261, V352, and V353, all with periods of about $\sim 0.4$ days $(\log P \sim-0.4)$. If their magnitudes are well constrained, then their periods might be consistent with second-overtone pulsations, in which case

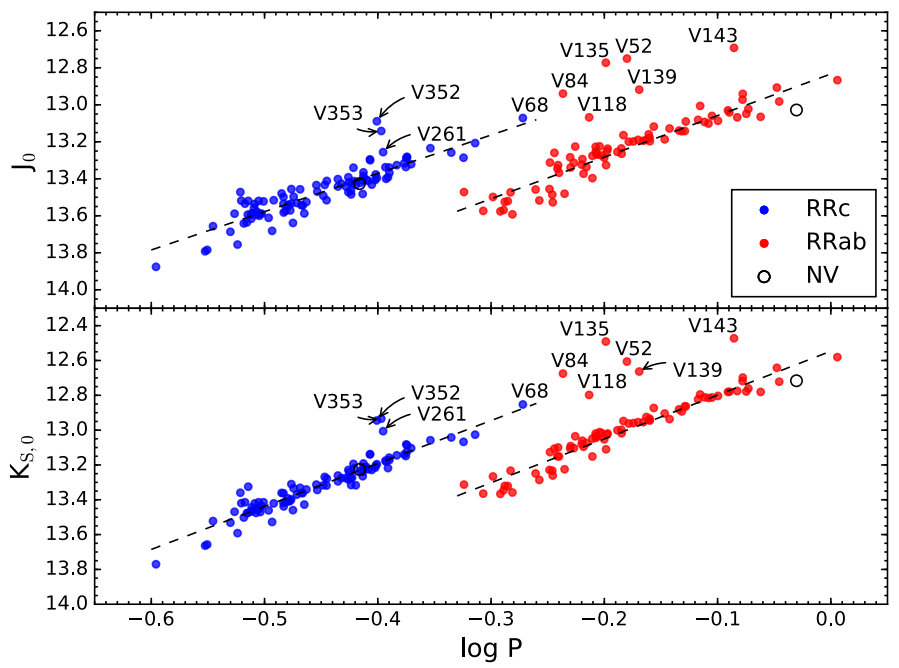

Fig. 2. De-reddened $J$ and $K_{\mathrm{S}}$ magnitudes versus period for RRLs in $\omega$ Cen. RRab stars are shown in red, and RRc stars are shown in blue. The dashed lines correspond to the best least-squares fits to the measurements, carried out separately for the $a b$ - and $c$-type RRL, once the labeled stars have been removed from the samples (see text).

they would be candidate RRe stars, for which one might expect $P_{2} / P_{0} \approx 0.57$ (e.g., Catelan \& Smith 2015). However, RRe stars are normally expected to be bluer than the RRc stars and to have short periods (Catelan \& Smith 2015, and references therein), whereas the long-period V261 and V353 appear redder instead, and fall near the RRab stars in the color-magnitude diagram (CMD; Navarrete et al. 2015b). V352 appears brighter than the RRc stars, but not bluer. It is perhaps more likely that these stars are affected by blends, and should accordingly be discarded from our analysis. As these three stars do not have previous metallicity measurements, they were considered neither in the derivation of empirical PL- $Z$ relations nor in the distance modulus calculation.

Table A.1 lists all the cluster member RRL stars with metallicity measurements from Rey et al. (2000) and/or Sollima et al. (2006a), including their equatorial coordinates, periods, $J$ and $K_{\mathrm{S}}$ intensity-averaged mean magnitudes and their corresponding (statistical) errors, subtypes, and metallicities. Since the number of RRL stars with spectroscopic metallicity is large, our sample allows us to obtain purely empirical PL $-Z$ relations both in $J$ and $K_{\mathrm{S}}$. To do this, we adopted the metallicities from Sollima et al. (2006a) and "fundamentalized" the periods of RRc stars using the relation $\log P_{0}=\log P_{1}+0.127$, corresponding to a period ratio $P_{1} / P_{0}=0.746$, as adopted by Del Principe et al. (2006). We prefer the metallicities from Sollima et al. (2006a) instead of those derived by Rey et al. (2000) because the former were derived based on high-resolution spectra. The metallicities were transformed into $\log Z$ using the relation

$\log Z=[\mathrm{Fe} / \mathrm{H}]+\log (0.362+0.638 f)+\log Z_{\odot}$ 


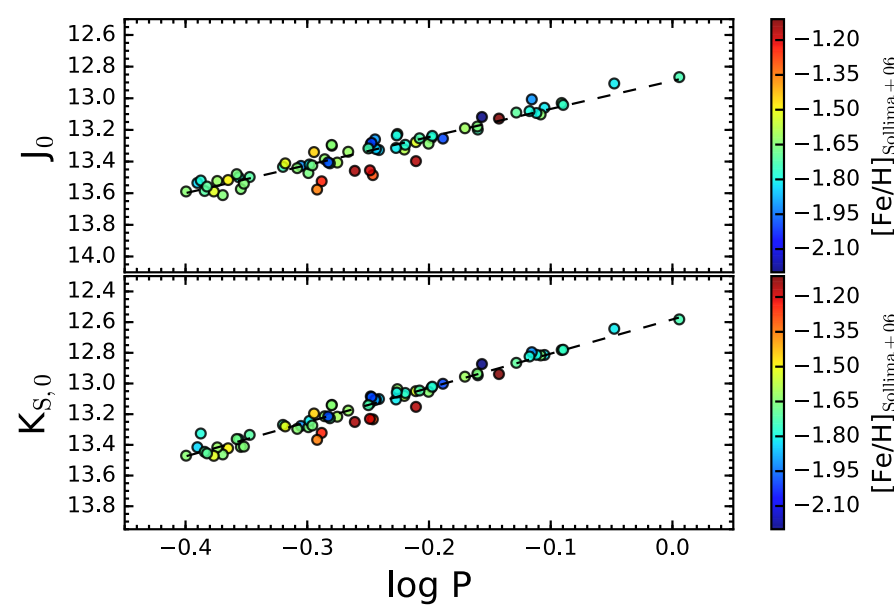

Fig. 3. Top panel: de-reddened $J$ magnitudes versus period with metallicities from Sollima et al. (2006a) in the color-bar. Bottom panel: the same as in the top panel, but for the $K_{\mathrm{S}}$ magnitude. Dashed lines are the empirical PL $-Z$ relations derived from our data set, evaluated at the mean RR Lyrae $[\mathrm{Fe} / \mathrm{H}]=-1.67 \mathrm{dex}$.

Table 2. Empirical PL $-Z$ relations for RRL stars in $\omega$ Cen.

\begin{tabular}{ccccc}
\hline \hline Band & $a$ & $b$ & $c$ & $R^{2}$ \\
\hline \multicolumn{5}{c}{$m_{X}=a \log P+b[\mathrm{Fe} / \mathrm{H}]+c$} \\
\hline$J$ & $-1.774 \pm 0.061$ & $0.153 \pm 0.027$ & $13.079 \pm 0.075$ & 0.936 \\
$K_{\mathrm{S}}$ & $-2.232 \pm 0.044$ & $0.141 \pm 0.020$ & $12.752 \pm 0.054$ & 0.985 \\
\hline
\end{tabular}

Notes. The relation has the form $m_{X}=a \log P+b[\mathrm{Fe} / \mathrm{H}]+c$, where $X$ corresponds to the bandpass.

(Salaris et al. 1993), where the $\alpha$-element enhancement factor $f=10^{[\alpha / \mathrm{Fe}]}$. For consistency with Catelan et al. (2004), $Z_{\odot}=0.017$ and $f=3$ were adopted. The coefficients and one standard deviation error of the least-squares fit are reported in Table 2. Figure 3 displays the sample used and the PL-Z relations obtained both for $J$ and $K_{\mathrm{S}}$, evaluated at the mean metallicity of the RRLs, $[\mathrm{Fe} / \mathrm{H}]=-1.67 \mathrm{dex}$. As can be seen from the plot, the $J$-band relation presents more scatter than the $K_{\mathrm{S}}$ one, which is consistent with theoretical expectations (e.g., Catelan et al. 2004) and with the fact that the $J$-band light curves present higher amplitudes, which can induce more scatter in the corresponding average quantities. The value of the $J$-band $\log P$ slope $a$ is in excellent agreement with the theoretical predictions, whereas there is a small disagreement in the case of $K_{\mathrm{S}}$, with the predicted value of $a$ being larger (in absolute value) by about 0.1 (see Table 3 in Coppola et al. 2011). As far as the metallicity dependence is concerned, our derived $b$ slopes are significantly steeper than was reported in Sollima et al. (2006b), but only slightly steeper than predicted by the Catelan et al. (2004) models. Naturally, we anticipate that the Gaia mission (Clementini et al. 2016) will provide the accurate distances to RR Lyrae stars that are needed in order to establish the definitive slopes and zero points of the PL- $Z$ relations. In the meantime, however, considering the reasonable agreement between the Catelan et al. (2004) model predictions and our results, we will employ the former in the remainder of our analysis.

In order to estimate the distance modulus of the cluster based on RRL stars, the calibrated theoretical PL-Z relations in the
Table 3. Distance moduli based on RRLs using spectroscopic metallicities and different pulsational modes.

\begin{tabular}{lcc}
\hline \hline & $\begin{array}{c}\left(J-M_{J}\right)_{0} \\
(\mathrm{mag})\end{array}$ & $\begin{array}{c}\left(K_{\mathrm{S}}-M_{K_{\mathrm{S}}}\right)_{0} \\
(\mathrm{mag})\end{array}$ \\
\hline \multicolumn{3}{c}{ Metallicities from Rey et al. (2000) } \\
\hline RRab (64 stars) & $13.728 \pm 0.063$ & $13.752 \pm 0.043$ \\
RRc (53 stars) & $13.685 \pm 0.058$ & $13.709 \pm 0.047$ \\
RRab+RRc (117 stars) & $13.698 \pm 0.064$ & $13.722 \pm 0.049$ \\
\hline \multicolumn{3}{c}{ Metallicities from Sollima et al. (2006) } \\
\hline RRab (33 stars) & $13.706 \pm 0.042$ & $13.746 \pm 0.027$ \\
RRc (31 stars) & $13.693 \pm 0.047$ & $13.728 \pm 0.040$ \\
RRab+RRc (64 stars) & $13.698 \pm 0.046$ & $13.735 \pm 0.036$ \\
\hline
\end{tabular}

VISTA filter system for the $J$ and $K_{\mathrm{S}}$ bands (Eqs. (1) and (3) in Alonso-García et al. 2015, which were adapted from Catelan et al. 2004) were used. PL relations for RRL stars have a nonnegligible dependence on metallicity, even though the dependence is lower in the near-IR bands compared to the optical (e.g., Catelan et al. 2004). Using the metallicities spectroscopically derived for RRL stars in $\omega$ Cen by Rey et al. (2000) and Sollima et al. (2006a), the distance modulus for the cluster was calculated using: (1) only the RRab stars; (2) only the RRc stars; and (3) all RRab and RRc stars. The periods of RRc stars were fundamentalized, as noted previously. $[\mathrm{Fe} / \mathrm{H}]$ values for 64 and $53 \mathrm{RRab}$ and RRc stars, respectively, are available in the cata$\log$ of Rey et al. (2000), while from Sollima et al. (2006a) the metallicities of $33 \mathrm{RRab}$ and also $31 \mathrm{RRc}$ stars were considered. Seven RRL from Rey et al. and four from Sollima et al. were not considered as they do not have reported errors in the metallicities, those measurements being highly uncertain. Both metallicity catalogs were compared by Sollima et al., who found a systematic offset of $\Delta[\mathrm{Fe} / \mathrm{H}]$ (Sollima et al. - Rey et al.) $=-0.06$ dex, with a dispersion of 0.3 dex. Because of that, the two metallicity sources were treated separately. The weighted-average distance modulus found with the $J$ and $K_{\mathrm{S}}$ magnitudes of the individual RRLs in the three different cases are listed in Table 3.

Figure 4 shows the magnitude differences between the mean $J$ (upper panels) and $K_{\mathrm{S}}$ (bottom panels) magnitudes and the derived PL $-Z$ relations (listed in Table 3 ), as a function of the metallicity from Sollima et al. (2006a) and Rey et al. (2000). As expected, there is less scatter for the $K_{\mathrm{S}}$ magnitudes (right panels), compared to the residuals for the $J$ band (left panels). The $3 \sigma$ residuals are $\sim 0.13$ and $0.10 \mathrm{mag}$ for $J$ and $K_{\mathrm{S}}$, respectively, considering the metallicities derived by Sollima et al. (2006a). When the metallicities of Rey et al. (2000) are adopted, the $3 \sigma$ residuals are slightly larger, with 0.19 and 0.13 mag for $J$ and $K_{\mathrm{S}}$ magnitudes, respectively. For both $J$ and $K_{\mathrm{S}}$, the residuals as a function of the $[\mathrm{Fe} / \mathrm{H}]$ values derived by Sollima et al. (2006a) are clustered around zero, and there is no evident trend with metallicity, proving that the metallicity term in the derived PL $-Z$ relations is well represented by the fit. Nonetheless, for the metallicities derived by Rey et al. (2000), there seems to be a small correlation between metallicities and magnitudes. However, we emphasize that our relations (Table 2) were derived using the metallicities from Sollima et al. (2006a), who used highresolution spectroscopy in their work, presumably leading to more accurate values than in the case of the $\Delta S$ method used by Rey et al. (2000). 

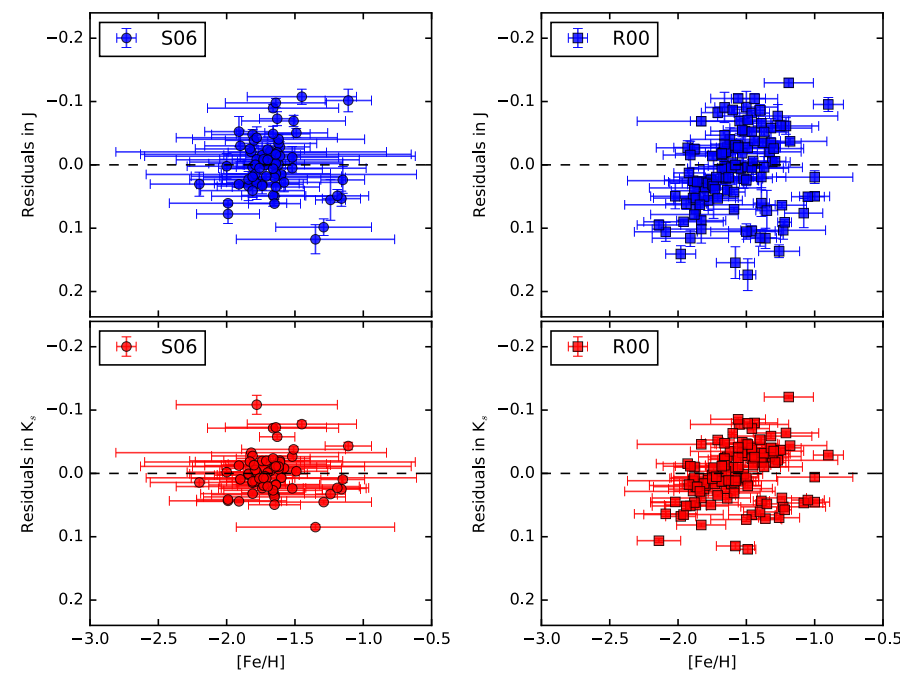

Fig. 4. Difference between the observed mean magnitudes and the derived PL- $Z$ relations as a function of metallicity. Top panels: show the residuals for $J$, while the residuals in $K_{\mathrm{S}}$ are shown in the bottom panels. Left and right panels: correspond to the metallicities derived by Sollima et al. (2006a) and Rey et al. (2000), respectively.

\subsection{Anomalous Cepheids}

Figure 2 shows that one RRab star, namely V84, is brighter than any other RRab that follows the PL relation, reaching the region where the RRc stars are located. However, the position in the CMD is in agreement with a cluster member star (Navarrete et al. 2015b). The period was first derived by Bailey (1902) and confirmed by the $J$ and $K_{\mathrm{S}}$ light curves, discarding an RRc classification. However, Sandage (1990), Longmore et al. (1990), and Nemec et al. (1994) all proposed that V84 and also V15, V68 (the longest-period RRc star in the cluster), and V99 could be ACEPs, rather than RRL stars.

Sparse measurements in the near-IR have been done for ACEPs. Only recently, Ripepi et al. (2014a) presented a large catalog of ACEPs in the Large Magellanic Cloud which have been gathered using near-IR observations performed by the VMC (Cioni et al. 2011).

Figure 5 shows the PL relation for the ACEPs at the $\omega$ Cen distance using the calibrated PL relation derived by Ripepi et al. (2014a), and adopting $\mu_{0}=13.708 \mathrm{mag}$ as $\omega$ Cen's true distance modulus (see Sect. 4). The PL relation is shown as a dotted line and the dashed lines above and below it represent $\pm 0.1 \mathrm{mag}$ deviations.

As can be noted, between $\log P \sim-0.35$ and $\sim-0.18$, the ACEPs and RRc PL relations cover a similar region of magnitudes, which does not allow us to cleanly separate both types of stars based on this diagram if they are both at the same distance. Thus, using the magnitudes from VISTA it is not possible to confirm or to reject the possibility that V68 and V84 are ACEPs in $\omega$ Cen. If V84 is not an ACEP, it should be a foreground RRab. V15 and V99, the other RRab stars proposed as ACEP candidates, follow closely the RRab PL relation, as can also be seen in Fig. 3, discarding the possibility of being ACEP variables.

\subsection{SX Phoenicis}

The $\omega$ Cen field contains a large number of SX Phoenicis stars larger, in fact, than for any other GC - thus providing an excellent opportunity to calibrate the SX Phe PL relation. Previous such studies have, however, been limited to the optical.

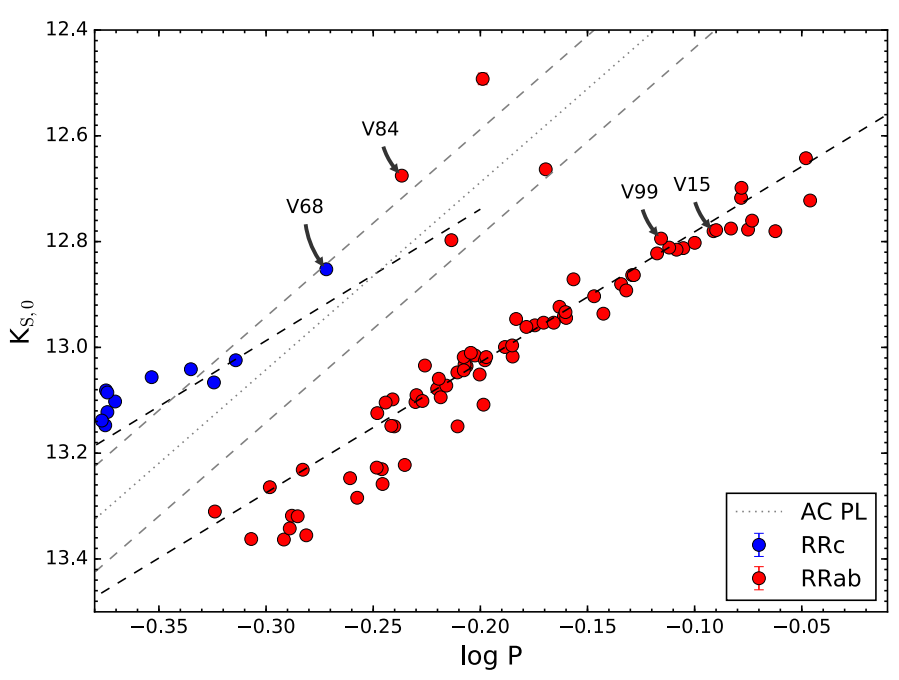

Fig. 5. De-reddened $K_{\mathrm{S}}$ magnitudes versus period around the locations of V68 and V84, two candidate ACEPs in $\omega$ Cen. The mean trend of $\mathrm{RRab}$ and RRc are denoted by the black dashed lines, whereas the dotted line shows the $K_{\mathrm{S}}$-PL relation for ACEPs, as derived by Ripepi et al. (2014a), shifted to the adopted distance modulus of $\mu_{0}=13.708$ mag. The gray dashed lines represent \pm 0.1 mag deviations around the latter relation.

Nemec et al. (1994) considered the $B$ and $V$ magnitudes of three SX Phe stars from $\omega$ Cen as well as 11 more from other GCs in order to derive observational PL relations. McNamara (2011) considered most of the known SX Phe in $\omega$ Cen (not including the five SX Phe stars from Weldrake et al. 2007), finding a high spread in the $\log P-\langle V\rangle$ diagram (see his Fig. 9), which may be partially explained by the variation of metallicity values in the cluster stars. Nevertheless, there are no previous metallicity measurements for the SX Phe stars in the cluster. According to McNamara (2011), the scatter can be reduced by selecting a subsample of SX Phe stars with long periods $(\log P \geq-1.47)$, which he argues are likely to be metal-poor $([\mathrm{Fe} / \mathrm{H}] \leq-1.0)$, fundamental-mode pulsators.

When plotting the $\log P-J$ and $-K_{\mathrm{S}}$ diagrams, the same situation as in McNamara (2011; i.e., high spread) is found, as can be see in Fig. 6. The field SX Phe stars, namely the foreground star V65 and the background stars V297 and ID-92 from Weldrake et al. (2007), were not included in the diagram.

The large amount of scatter notwithstanding, Fig. 6 also reveals the presence of a tight "lower envelope" of stars, below which only a handful of faint stragglers are found. We conjecture that this may correspond to the sequence of fundamental-mode pulsators in the cluster. The five stars located below this lower envelope (V302, V307, V315, V323, and V324) are probably non-radial pulsators. The three stars with the shortest-periods (V294, V295, and V296, with $\log P<-1.6)$ are likely highovertone pulsators. The longest-period star, V328, is considered a fundamental-mode pulsator (Olech et al. 2005), but its magnitudes in the visible and in the $J$ and $K_{\mathrm{S}}$ bands are fainter than the linear trend that the other stars at shorter periods define. Because of that, it will also be excluded to derive the fundamental-mode PL relation for $S X$ Phe stars.

Table A.2 presents the variable ID, equatorial coordinates, periods, and intensity-averaged $J$ and $K_{\mathrm{S}}$ magnitudes for the 45 SX Phe fundamental-mode candidates. The stars that exhibit light curves with clear evidence of variability are explicitly tagged as "Variability recovered" in this table. Figure 7 shows the thus selected 45 fundamental-mode candidates, in which 


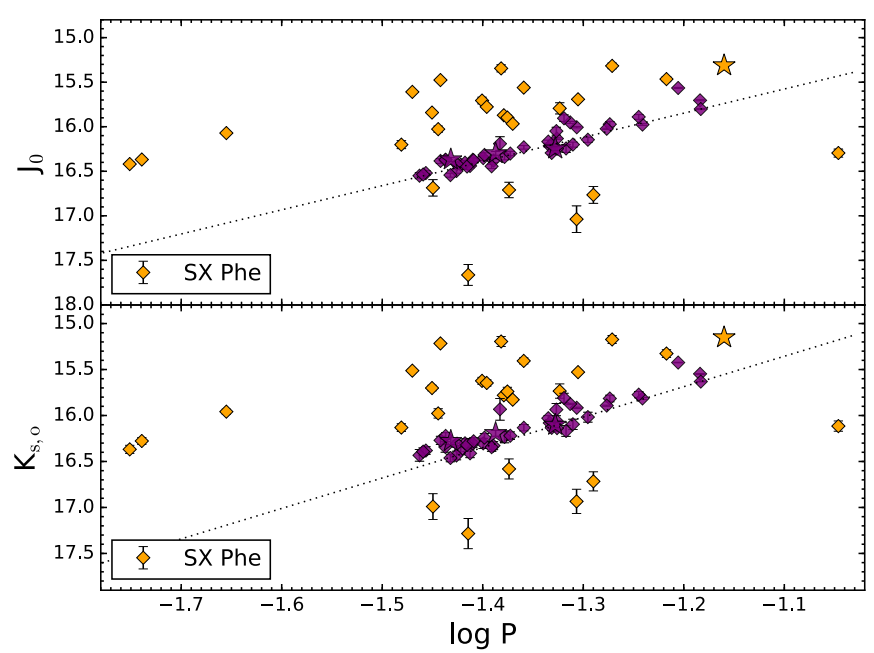

Fig. 6. De-reddened $J$ and $K_{\mathrm{S}}$ magnitudes versus period for all the SX Phe stars that appear to be members of the cluster. Those from Kaluzny et al. (2004) are plotted as orange diamonds while the star symbols denote those discovered by Weldrake et al. (2007). The "lowerenvelope" stars that tentatively follow a common trend and would correspond to fundamental-mode pulsators are shown in purple.

three of the four SX Phe stars from Weldrake et al. (2007) are included. In Fig. 7, the dotted lines represent the least-squares best fit to the fundamental-mode candidates, while the dashed lines are the expected PL relation for first-overtone pulsators, assuming that both relations are parallel and adopting a ratio of the first-overtone period to the fundamental-mode period of 0.775 (McNamara 2011), which implies a magnitude shift (at fixed $\log P$ ) of 0.26 and $0.37 \mathrm{mag}$ for $J$ and $K_{\mathrm{S}}$, respectively.

Based on these 45 selected SX Phe stars, observational PL relations for the fundamental mode candidates obtained are

$$
\begin{aligned}
& J_{0}=-(3.04 \pm 0.17) \log P+(12.10 \pm 0.22) \\
& \sigma=0.09 \mathrm{mag}, \\
& K_{\mathrm{S}, 0}=-(3.39 \pm 0.24) \log P+(11.51 \pm 0.30) \\
& \sigma=0.11 \mathrm{mag} .
\end{aligned}
$$

The dispersion around both these relations are quite similar and smaller than that associated to the $V$-band relation derived by McNamara (2011), being the latter 0.13 mag. This difference is mainly due to the intrinsic dispersion of the optical PL relations compared to the near-IR for any type of pulsating variable star (Catelan \& Smith 2015). Despite the fact that most of the light curves of the SX Phe stars could not be fully recovered, their behavior in the $\log P$-magnitude diagram is consistent with most of them pulsating in the fundamental mode.

However, according to Olech et al. (2005), there are some first-overtone SX Phe among the ones that we have considered as fundamental-mode pulsators. Figure 8 shows the same stars as Fig. 7 but now the fundamental-mode and first-overtone candidates as derived by Olech et al. are plotted as purple circles and yellow triangles, respectively. The diagram includes the four SX Phe (members of the cluster) discovered by Weldrake et al., after the study of Olech et al. was published (open stars).

From the figure, the SX Phe found by Weldrake et al. (2007) are consistent with three of them pulsating in the fundamental mode. On the other hand, the one with the longest period, namely ID-7 (at $\log P \sim-1.16$ ), appears as a bona fide first-overtone pulsator. The other SX Phe stars, despite being considered firstovertone pulsators by Olech et al. (2005), follow the PL relations

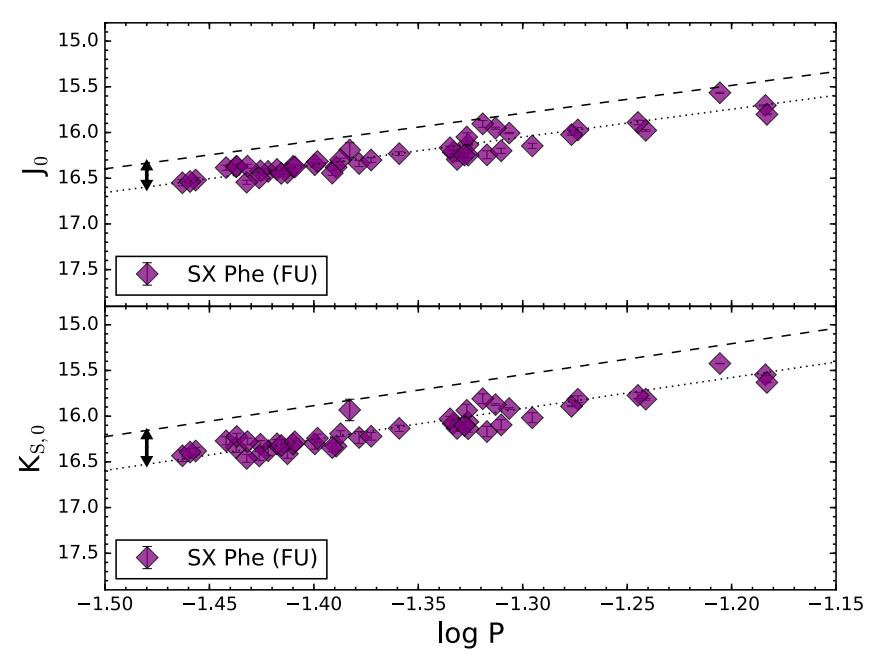

Fig. 7. Fundamental-mode SX Phe candidates from which observational $J_{0}$ and $K_{\mathrm{S}, 0} \mathrm{PL}$ relations were derived. The dotted lines correspond to the best least-squares fit associated to each filter. The dashed lines correspond to the expected position of the first-overtone pulsators, assuming that fundamental and first-overtone PL relations are parallel.

as given by Eqs. (2) and (3), suggesting that they too may be fundamental-mode pulsators.

\section{On the $\omega$ Cen pulsational distance modulus}

\subsection{Systematic errors}

Before we adopt a pulsational distance modulus for $\omega$ Cen, based on the different values obtained using T2Cs and RRL stars, the different sources of systematic errors will be evaluated.

In the case of $\mathrm{T} 2 \mathrm{Cs}$, one possible source of systematic error in the distance modulus values is that some first-overtone pulsators could be considered as pulsating in the fundamental mode. To estimate the error associated to this, we could consider the difference between the distance modulus obtained when different stars are used, as listed in Table 1. Adopting the $J$ and $K_{\mathrm{S}}$-based true distance moduli derived using W Vir and BL Her stars, the systematic errors should be at least 0.04 and 0.03 mag, respectively. The metallicity term could constitute an additional source of systematic errors but, given the low metallicity dependence of the T2C PL relation, we expect that these errors are lower than $0.01 \mathrm{mag}$, both in $J$ and in $K_{\mathrm{S}}$.

In the case of RRL stars, one source of systematic error could be provided by the sample choice, that is, including or not fundamental and first-overtone pulsators simultaneously in the fits. Del Principe et al. (2006) found, based on synthetic HB models, that the relations for fundamental and first-overtone RRL are not exactly parallel, and that the use of fundamentalized RRc periods produces an increase (by a factor of two) in the uncertainty of the zero points and slopes of their relations. Nevertheless, Catelan et al. (2004) still found tight near-IR PL relations, considering RRab and (fundamentalized) RRc at the same time.

Table 3 lists the three possible cases, that is, using only RRab, only RRc (with fundamentalized periods), and RRL stars from both pulsational modes (again with RRc periods fundamentalized). The metallicities derived by Sollima et al. (2006a) seem to be more accurate (i.e., close to the actual metallicity of the stars) than the ones derived by Rey et al., in the sense that more precise distance moduli are obtained. The fact that the results are always consistent (to within the errors), irrespective of the adopted subsample, suggests that RRab and (fundamentalized) 


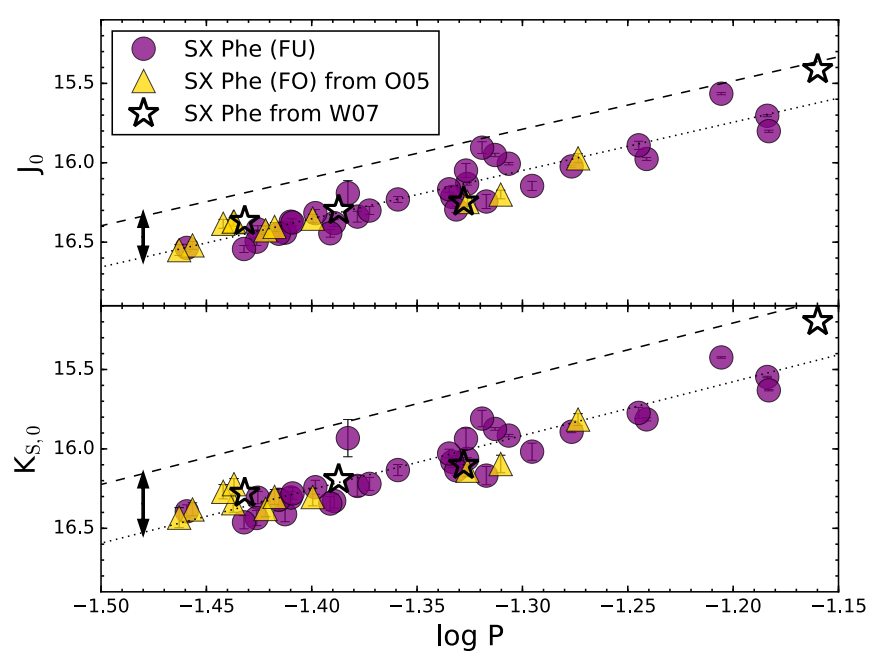

Fig. 8. Fundamental (in purple circles) and first-overtone (in yellow triangles) SX Phe as classified by Olech et al. (2005), based on their frequency spectrum. The dotted lines are the PL relations derived previously. As can be seen, there are some claimed first-overtone pulsators that also follow the relations defined by fundamental-mode pulsators.

Table 4. Adopted distance moduli based on T2Cs and RRL stars.

\begin{tabular}{lcccc}
\hline \hline & $\begin{array}{c}\mu_{0}(J) \\
(\mathrm{mag})\end{array}$ & $\begin{array}{c}\sigma_{\text {total }} \\
(\mathrm{mag})\end{array}$ & $\begin{array}{c}\mu_{0}\left(K_{\mathrm{S}}\right) \\
(\mathrm{mag})\end{array}$ & $\begin{array}{c}\sigma_{\text {total }} \\
(\mathrm{mag})\end{array}$ \\
\hline T2Cs & 13.675 & 0.042 & 13.652 & 0.031 \\
RRLs & 13.701 & 0.024 & 13.733 & 0.017 \\
\hline
\end{tabular}

RRc stars do indeed follow closely the same PL relation in the near-IR.

Comparison between the different distance moduli obtained using the two different sources of metallicity in Table 3 suggests to us that the metallicity scale can be one source of systematic error in the final derived distance modulus. Based on these results, we adopt a value of 0.02 and $0.01 \mathrm{mag}$ for $J$ and $K_{\mathrm{S}}$ respectively, as representative of the main identified sources of systematic errors affecting the $\omega$ Cen distance modulus, as derived on the basis of the near-IR PL relations for RRL stars. Moreover, the individual metallicities for RRL stars have errors of the order of $\sim 0.19$ and 0.31 dex, as reported by Rey et al. (2000) and Sollima et al. (2006a), respectively. These uncertainties, propagated into the PL- $Z$ relations used to derive the distance modulus, add the following errors: 0.04 and $0.03 \mathrm{mag}$ for $J$ and $K_{\mathrm{S}}$ distance modulus, with metallicities from Rey et al., and 0.06 and $0.05 \mathrm{mag}$ for the $J$ and $K_{\mathrm{S}}$ values derived with the metallicities from Sollima et al. At the same time, results from previous studies (e.g., Cacciari \& Clementini 2003; Del Principe et al. 2006; Feast et al. 2008; Gran et al. 2015) suggest that distances derived on the basis of such relations can probably not yet be considered accurate to better than about $\pm 0.1 \mathrm{mag}$. Thus, we adopt the latter value as a conservative estimate of the systematic error affecting the distance moduli provided in our study.

\subsection{Pulsational distance modulus from T2Cs and RRL stars}

Table 4 lists the weighted-average distance modulus values from T2Cs and RRL stars and sigma as the dispersion around the mean. The weights for each individual distance modulus values were defined as the inverse of the squared of the total error, including statistical and systematic errors (summed up in quadrature). As expected, the error is lower for the $K_{\mathrm{S}}-\mathrm{PL}$ relations compared to $J$, since the former have lower intrinsic dispersion, and is based on a more extensive dataset. Comparing both variability types, T2Cs lead to a less accurate distance modulus, probably because they are few in number, which increases the statistical error, and they have higher systematic errors since the effect of metallicity and pulsation mode separation in the derivation of the PL relations is not completely established.

Considering the distance modulus values found using T2Cs stars as well as RRL stars, both in $J$ and $K_{\mathrm{S}}$, the weightedaverage distance modulus to $\omega$ Cen was determined, leading to a final value of $\mu_{0}=13.708 \pm 0.035 \mathrm{mag}$, where the error bar corresponds to the standard deviation of the mean. Given the discussion of systematic errors in the previous section, our final adopted distance modulus is $\mu_{0}=13.708 \pm 0.035 \pm 0.10 \mathrm{mag}$, where the error bars correspond to the adopted statistic and systematic errors, respectively. Adding the errors in quadrature (as recommended, for instance, by Barlow et al. 2002), this corresponds to a heliocentric distance of $5.52 \pm 0.27 \mathrm{kpc}$.

As a further check, we have also explored the results of Feast (2011), who suggested that T2Cs and RRL follow basically the same PL relation in $K$. Using metallicities for the T2Cs from Gonzalez \& Wallerstein (1994) and van Loon et al. (2007), and assuming that the same near-IR relation as for the RRL applies also to the T2Cs, we obtain for the latter a distance modulus of $\mu_{0}=13.70 \pm 0.04 \mathrm{mag}$ (standard deviation of the mean), in excellent agreement with the previously derived value. On the other hand, carrying out the same analysis using the $J$-band data does not provide similarly consistent results, with a resulting distance modulus that is shorter by about $0.3 \mathrm{mag}$. This suggests that the T2Cs and RRL stars may indeed follow the same PL relation in $K_{\mathrm{S}}$, but not in $J$.

Table 5 lists some of the true distance modulus values for $\omega$ Centauri found in the literature. In order to put all of them in the same system, the color excess was set as $E(B-V)=$ $0.12 \mathrm{mag}$, a standard extinction law with $R_{V}=3.1$ was adopted, and the apparent $V$ distance moduli were converted accordingly. The work of Caputo et al. (2002) listed some of $\omega$ Cen's distance moduli derived in the literature and compared them, considering the influence of the metallicity spread as well as the possible helium enhancement associated to second-generation stars in the cluster. Distance modulus values based on PL-HB type relations were derived for different $\alpha$-enhancement levels by Del Principe et al. (2006), and compared with other values from the literature.

The distance modulus found in this work appears in excellent agreement with most of the values listed in Table 5. The distance modulus derived by Del Principe et al. (2006), using a combination of near-IR light curves and 2MASS single-epoch magnitudes for different RRL stars in the cluster, appears quite similar to the average value found using our fully phase-folded light curves derived from VISTA filters.

\subsection{Difference between T2C- and RRL-based distance moduli}

The $\omega$ Cen distance modulus values derived using T2Cs appear shorter than those found with the RRL stars, by an amount ranging from $0.03 \mathrm{mag}$ in $J$ to $0.08 \mathrm{mag}$ in $K_{\mathrm{S}}$ (see Table 4). Such a difference could not be explained by the metallicity term in the T2Cs PL relations as, if included, the corresponding distance moduli would be even lower. 
Table 5. Distance modulus for $\omega$ Cen from the literature.

\begin{tabular}{|c|c|c|}
\hline Method & $\mu_{0}(\mathrm{mag})^{a}$ & Reference \\
\hline$B$ and $V$ PL relations for Pop. II stars & $13.53 \pm 0.20$ & Nemec et al. (1994) \\
\hline High-amplitude $\delta$ Sct stars & $14.05 \pm 0.10$ & $\operatorname{McNamara}(2000)^{b}$ \\
\hline Detached eclipsing binary (V212) & $13.68 \pm 0.11$ & Thompson et al. (2001) \\
\hline Detached eclipsing binary (V212) & $13.72 \pm 0.04$ & Kaluzny et al. (2002) \\
\hline$A_{V}-\log P$ models for RRab stars & $13.64 \pm 0.11$ & Caputo et al. (2002) \\
\hline$M_{V}-\log P$ models for RRc stars & $13.71 \pm 0.11$ & Caputo et al. (2002) \\
\hline$M_{V}-[\mathrm{Fe} / \mathrm{H}]$ relation (RRL stars) & $13.72 \pm 0.11$ & Del Principe et al. $(2006)^{c}$ \\
\hline$M_{V}-[\mathrm{Fe} / \mathrm{H}]$ relation (RRL stars) & $13.62 \pm 0.11$ & Del Principe et al. $(2006)^{d}$ \\
\hline $\mathrm{PL}-Z\left(K_{\mathrm{S}}\right)$ & $13.77 \pm 0.07$ & Del Principe et al. $(2006)^{e}$ \\
\hline $\mathrm{PL}-\tau_{\mathrm{HB}}\left(K_{\mathrm{S}}, f=3, \tau_{\mathrm{HB}}=0.940\right)$ & $13.69 \pm 0.06$ & Del Principe et al. (2006) \\
\hline $\mathrm{PL}-\tau_{\mathrm{HB}}\left(J, f=3, \tau_{\mathrm{HB}}=0.940\right)$ & $13.70 \pm 0.10$ & Del Principe et al. (2006) \\
\hline $\mathrm{PL}-\tau_{\mathrm{HB}}\left(K_{\mathrm{S}}, f=3, \tau_{\mathrm{HB}}=0.934\right)$ & $13.72 \pm 0.06$ & Del Principe et al. $(2006)^{f}$ \\
\hline $\operatorname{PL}-\tau_{\mathrm{HB}}\left(J, f=3, \tau_{\mathrm{HB}}=0.934\right)$ & $13.76 \pm 0.10$ & Del Principe et al. (2006) \\
\hline Cluster dynamics & $13.41 \pm 0.14$ & van de Ven et al. (2006) \\
\hline$M_{V}-[\mathrm{Fe} / \mathrm{H}]$ relation (RRL stars) & $13.68 \pm 0.27$ & Weldrake et al. (2007) \\
\hline SX Phe $\log P-V$ relation & $13.76 \pm 0.06$ & McNamara (2011) \\
\hline Optical Period-Wesenheit relations (RRL stars) & $13.71 \pm 0.08$ & Braga et al. (2016) \\
\hline Weighted average & $13.72 \pm 0.13$ & \\
\hline Weighted-average PL relations (T2Cs and RRLs) & $13.708 \pm 0.035$ & This work \\
\hline
\end{tabular}

Notes. ${ }^{(a)}$ Most of the error estimates do not consider the contribution of systematic errors, which in the case of T2Cs and RRL stars can reach values of order $\approx 0.10 \mathrm{mag} .{ }^{(b)}$ The listed error was determined considering the internal uncertainties of the mean distance modulus value, $\pm 0.02 \mathrm{mag}$, and the observational error, $\pm 0.1 \mathrm{mag}$, as described by McNamara (2000). ${ }^{(c)}$ Based on the $M_{V}-[\mathrm{Fe} / \mathrm{H}]$ relation from Bono et al. (2003) and mean $V$ magnitudes from Kaluzny et al. (2004). ${ }^{(d)}$ Based on the $M_{V}-[\mathrm{Fe} / \mathrm{H}]$ relation of Catelan et al. (2004) and $V$ magnitudes from Kaluzny et al. (2004). ${ }^{(e)}$ Based on the semi-empirical $J$-band PL-Z relation from Bono et al. (2003). ${ }^{(f)}$ Based on the PL- $\tau_{\mathrm{HB}}$ relations from Catelan et al. (2004).

One possible explanation for the difference could rest on the multiple populations in $\omega \mathrm{Cen}$ and the associated helium enhancement (Caputo et al. 2002; Sollima et al. 2006a; Marconi et al. 2011). He-rich stars are expected to populate the bluest part of the $\mathrm{HB}$, where the $\mathrm{T} 2 \mathrm{C}$ progenitors are thought to be located, thus those stars affect the magnitudes and pulsational periods of T2Cs with respect to the expected ones for stars with primordial helium abundance.

Nonetheless, Marconi et al. (2011) developed evolutionary and pulsational models in order to derive the number of variable stars in the HB of $\omega$ Cen considering different helium abundances. The authors compared the number fraction of T2Cs over the number of variable stars in the instability strip as predicted by the models and the observed one. For $Y=0.24,2 \%$ of T2Cs are found with the models, in good agreement with the observational $3 \%$. Helium enhancement is taken into account considering two stellar populations with primordial initial helium abundances ( $80 \%$ of the stars) and enhanced-helium $(Y=0.3$, $20 \%$ of the stars). It was found that the percentage of expected $\mathrm{T} 2 \mathrm{Cs}, 5 \%$, would be higher than the one actually observed. This suggests that T2C stars in $\omega$ Cen are predominantly not Heenhanced.

For RRL stars, the authors found that the minimum fundamentalized period predicted by the models is in good agreement with the observed values of 0.34 days when the primordial helium abundance is considered. At higher helium abundance values, the shortest fundamentalized period increases, which is not observed. This also suggests that RRL stars in $\omega$ Cen are not He-enhanced.

We thus conclude that other sources of systematic error will have to be investigated, in order to explain the origin of the difference in distance moduli obtained on the basis of the T2C and RRL near-IR PL relations.

\section{Conclusions}

We have presented the implications of our recent, extensive time-series photometry of $\omega$ Cen (Navarrete et al. 2015b) for the near-IR (VISTA $J$ and $K_{\mathrm{S}}$ ) PL relations of different types of pulsators along the "classical" instability strip. These include T2Cs, ACEPs, RRL, and SX Phe stars. For the T2Cs and RRL stars, for which the corresponding near-IR relations had previously been calibrated, we use the results to obtain a new distance modulus estimate for the cluster, $\mu_{0}=13.708 \pm 0.035 \pm$ $0.10 \mathrm{mag}$, where the error bars correspond to the adopted statistical and systematic errors, respectively. Adding the errors in quadrature, this is equivalent to a heliocentric distance of $5.52 \pm 0.27 \mathrm{kpc}$, which is in excellent agreement with previous results from the literature ${ }^{1}$. An offset between the T2C and RRLbased result, at the level of a few hundredths of a magnitude (with the T2Cs implying a smaller distance), is however present, for reasons which are not completely clear at present.

The sizable number of RRL stars in the cluster allows us to derive new empirical near-IR PL-Z relations in the $J$ and $K_{\mathrm{S}}$ bandpasses. Adopting as the true distance modulus of the cluster $\mu_{0}=13.708 \mathrm{mag}$, the PL-Z relations presented in Table 2, in

\footnotetext{
1 After we submitted our paper, Bhardwaj et al. (2017) published new near-IR PL and Period-Wesenheit relations for T2Cs, using template-fit $I$ and $K_{\mathrm{S}}$ light curves. Combining both relations, the authors derived a true distance modulus for $\omega$ Cen of $\mu\left(K_{\mathrm{S}}\right)=13.70 \pm 0.11 \mathrm{mag}$, in good agreement with our derived distance modulus based on RRL stars, but slightly different from the distance modulus derived by Matsunaga et al. (2006) using T2Cs and the $K_{\mathrm{S}}$-band relation.
} 
terms of the absolute magnitude, are given by

$$
\begin{aligned}
M_{J}(\mathrm{RRL})=- & (1.77 \pm 0.06) \log P \\
& +(0.15 \pm 0.03)[\mathrm{Fe} / \mathrm{H}]-(0.63 \pm 0.08), \\
M_{K_{\mathrm{S}}}(\mathrm{RRL})=- & (2.23 \pm 0.04) \log P \\
& +(0.14 \pm 0.02)[\mathrm{Fe} / \mathrm{H}]-(0.96 \pm 0.05) .
\end{aligned}
$$

For the ACEPs, we show that their expected positions in the near-IR PL relations are very similar to those of RRc stars. This makes it difficult to properly establish their pulsation status on the basis of our near-IR data alone.

For the SX Phe stars, we provide, for the first time, a calibration of their near-IR PL relation. Using a true distance modulus of $\mu_{0}=13.708 \mathrm{mag}$ for the cluster, and combining with Eqs. (2) and (3), we obtain

$$
\begin{aligned}
M_{J}(\mathrm{SX} P h e) & =-(3.04 \pm 0.17) \log P-(1.60 \pm 0.22), \\
M_{K_{\mathrm{S}}}(\mathrm{SX} \text { Phe }) & =-(3.39 \pm 0.24) \log P-(2.19 \pm 0.30)
\end{aligned}
$$

In the next (and final) paper of this series (Navarrete et al., in prep.), we will present our full near-IR catalog of variable stars in the $\omega$ Cen field.

Acknowledgements. We warmly thank the referee, B. Madore, for very usefu suggestions that helped to improve our paper. Support for this project is provided by the Ministry for the Economy, Development, and Tourism's Millennium Science Initiative through grant IC 120009, awarded to the Millennium Institute of Astrophysics (MAS); by the Basal Center for Astrophysics and Associated Technologies (CATA) through grant PFB-06/2007; by CONICYT's PCI program through grant DPI20140066; and by FONDECYT grants \#1141141 and \#1171273 (C.N., M.C.), \#1130196 (D.M.), \#1150345 (F.G.), \#3130320 (R.C.R.), and \#11150916 (J.A.-G.). C.N. acknowledges additional support from CONICYT-PCHA/Doctorado Nacional 2015-21151643. I.D. was supported by Sonderforschungsbereich SFB 881 "The Milky Way System" (subproject A3) of the German Research Foundation (DFG).

\section{References}

Alcock, C., Allsman, R. A., Alves, D. R., et al. 2000, ApJ, 536, 798 Alonso-García, J., Mateo, M., Sen, B., et al. 2012, AJ, 143, 70 Alonso-García, J., Dékány, I., Catelan, M., et al. 2015, AJ, 149, 99 Altmann, M., Catelan, M., \& Zoccali, M. 2005, A\&A, 439, L5 Anderson, J., \& van der Marel, R. P. 2010, ApJ, 710, 1032

Angeloni, R., Contreras Ramos, R., Catelan, M., et al. 2014, A\&A, 567, A100 Bailey, S. I. 1902, Annals of Harvard College Observatory, 38, 1

Barlow, R., Cahn, R., Cowan, G., et al. 2002, BABAR Analysis Document, 318, 1

Bedin, L. R., Piotto, G., Anderson, J., et al. 2004, ApJ, 605, L125

Bekki, K., \& Norris, J. E. 2006, ApJ, 637, L109

Bhardwaj, A., Macri, L. M., Rejkuba, M., et al. 2017, AJ, 153, 154

Bono, G., Caputo, F., Castellani, V., et al. 2003, MNRAS, 344, 1097

Braga, V. F., Stetson, P. B., Bono, G., et al. 2016, AJ, 152, 170

Cacciari, C., \& Clementini, G. 2003, in Stellar Candles for the Extragalactic

Distance Scale, eds. D. Alloin, \& W. Gieren (Berlin: Springer Verlag), Lect. Notes Phys., ASP Conf. Ser., 635, 105

Caputo, F., degl'Innocenti, S., \& Marconi, M. 2002, in Omega Centauri, A Unique Window into Astrophysics, eds. F. van Leeuwen, J. D. Hughes, \& G. Piotto, ASP Conf. Ser., 265, 185

Catelan, M., \& Smith, H. A. 2015, Pulsating Stars (Wiley-VCH)

Catelan, M., Pritzl, B. J., \& Smith, H. A. 2004, ApJS, 154, 633

Catelan, M., Minniti, D., Lucas, P. W., et al. 2011, in RR Lyrae Stars, Metal-Poor Stars, and the Galaxy, ed. A. McWilliam, Carnegie Observatory Series, 5, 145 Catelan, M., Dékány, I., Hempel, M., \& Minniti, D. 2013a, BAAA, 56, 153

Catelan, M., Minniti, D., Lucas, P. W., et al. 2013b, in 40 Years of Variable Stars: A Celebration of Contributions by Horace A. Smith, eds. K. Kinemuchi et al., 141

Cioni, M.-R. L., Clementini, G., Girardi, L., et al. 2011, A\&A, 527, A116

Clement, C. M., Muzzin, A., Dufton, Q., et al. 2001, AJ, 122, 2587

Clementini, G., Ripepi, V., Leccia, S., et al. 2016, A\&A, 595, A133
Cohen, R. E., \& Sarajedini, A. 2012, MNRAS, 419, 342

Coppola, G., Dall'Ora, M., Ripepi, V., et al. 2011, MNRAS, 416, 1056 Da Costa, G. S., \& Coleman, M. G. 2008, AJ, 136, 506

Dalton, G. B., Caldwell, M., Ward, A. K., et al. 2006, in SPIE Conf. Ser., 6269

Del Principe, M., Piersimoni, A. M., Storm, J., et al. 2006, ApJ, 652, 362

Di Criscienzo, M., Caputo, F., Marconi, M., \& Cassisi, S. 2007, A\&A, 471, 893 Dinescu, D. I. 2002, in Omega Centauri, A Unique Window into Astrophysics, eds. F. van Leeuwen, J. D. Hughes, \& G. Piotto, ASP Conf. Ser., 265, 365

D'Souza, R., \& Rix, H.-W. 2013, MNRAS, 429, 1887

Dupree, A. K., \& Avrett, E. H. 2013, ApJ, 773, L28

Dupree, A. K., Strader, J., \& Smith, G. H. 2011, ApJ, 728, 155

Emerson, J., \& Sutherland, W. 2010, The Messenger, 139, 2

Feast, M. 2011, in RR Lyrae Stars, Metal-Poor Stars, and the Galaxy, ed. A. McWilliam, Carnegie Observatory Series, 5, 170

Feast, M. W., Laney, C. D., Kinman, T. D., van Leeuwen, F., \& Whitelock, P. A 2008, MNRAS, 386, 2115

Fernández-Trincado, J. G., Robin, A. C., Vieira, K., et al. 2015, A\&A, 583, A76 Gonzalez, G., \& Wallerstein, G. 1994, AJ, 108, 1325

Gran, F., Minniti, D., Saito, R. K., et al. 2015, A\&A, 575, A114

Harris, W. E. 1996, AJ, 112, 1487

Harris, W. E. 2010, ArXiv e-prints [arXiv: 1012 . 3224]

Johnson, C. I., Pilachowski, C. A., Michael Rich, R., \& Fulbright, J. P. 2009, ApJ, 698, 2048

Joo, S.-J., \& Lee, Y.-W. 2013, ApJ, 762, 36

Kaluzny, J., Thompson, I., Krzeminski, W., et al. 2002, in Omega Centauri, A Unique Window into Astrophysics, ed. F. van Leeuwen, J. D. Hughes, \& G. Piotto, ASP Conf. Ser., 265, 155

Kaluzny, J., Olech, A., Thompson, I. B., et al. 2004, A\&A, 424, 1101

Klein, C. R., \& Bloom, J. S. 2014, ArXiv e-print [arXiv: 1404 .4870]

Klein, C. R., Richards, J. W., Butler, N. R., \& Bloom, J. S. 2014, MNRAS, 440, L96

Longmore, A. J., Fernley, J. A., \& Jameson, R. F. 1986, MNRAS, 220, 279

Longmore, A. J., Dixon, R., Skillen, I., Jameson, R. F., \& Fernley, J. A. 1990 MNRAS, 247, 684

Marconi, M., Bono, G., Caputo, F., et al. 2011, ApJ, 738, 111

Matsunaga, N., Fukushi, H., Nakada, Y., et al. 2006, MNRAS, 370, 1979

McGonegal, R., McAlary, C. W., McLaren, R. A., \& Madore, B. F. 1983, ApJ, 269, 641

McNamara, D. H. 1995, AJ, 109, 1751

McNamara, D. H. 2000, PASP, 112, 1096

McNamara, D. H. 2011, AJ, 142, 110

Meylan, G., Sarajedini, A., Jablonka, P., et al. 2001, AJ, 122, 830

Meza, A., Navarro, J. F., Abadi, M. G., \& Steinmetz, M. 2005, MNRAS, 359, 93

Minniti, D., Lucas, P. W., Emerson, J. P., et al. 2010, New Astron., 15, 433

Navarrete, C., Catelan, M., Contreras Ramos, R., et al. 2013, IBVS, 6078, 1

Navarrete, C., Chanamé, J., Ramírez, I., et al. 2015a, ApJ, 808, 103

Navarrete, C., Contreras Ramos, R., Catelan, M., et al. 2015b, A\&A, 577, A99

Nemec, J. M., Nemec, A. F. L., \& Lutz, T. E. 1994, AJ, 108, 222

Norris, J. E. 2004, ApJ, 612, L25

Olech, A., Dziembowski, W. A., Pamyatnykh, A. A., et al. 2005, MNRAS, 363, 40

Petersen, J. O., \& Christensen-Dalsgaard, J. 1999, A\&A, 352, 547

Piotto, G., Villanova, S., Bedin, L. R., et al. 2005, ApJ, 621, 777

Rey, S.-C., Lee, Y.-W., Joo, J.-M., Walker, A., \& Baird, S. 2000, AJ, 119, 1824

Ripepi, V., Marconi, M., Moretti, M. I., et al. 2014a, MNRAS, 437, 2307

Ripepi, V., Moretti, M. I., Marconi, M., et al. 2015, MNRAS, 446, 3034

Ripepi, V., Marconi, M., Moretti, M. I., et al. 2016, ApJS, 224, 21

Saito, R. K., Hempel, M., Minniti, D., et al. 2012, A\&A, 537, A107

Salaris, M., Chieffi, A., \& Straniero, O. 1993, ApJ, 414, 580

Sandage, A. 1990, ApJ, 350, 603

Schechter, P. L., Mateo, M., \& Saha, A. 1993, PASP, 105, 1342

Sollima, A., Borissova, J., Catelan, M., et al. 2006a, ApJ, 640, L43

Sollima, A., Cacciari, C., \& Valenti, E. 2006b, MNRAS, 372, 1675

Stetson, P. B. 1987, PASP, 99, 191

Stetson, P. B. 1994, PASP, 106, 250

Tailo, M., Di Criscienzo, M., D’Antona, F., Caloi, V., \& Ventura, P. 2016, MNRAS, 457, 4525

Templeton, M., Basu, S., \& Demarque, P. 2002, ApJ, 576, 963

Thompson, I. B., Kaluzny, J., Pych, W., et al. 2001, AJ, 121, 3089

Valcarce, A. A. R., \& Catelan, M. 2011, A\&A, 533, A120

van der Marel, R. P., \& Anderson, J. 2010, ApJ, 710, 1063

van de Ven, G., van den Bosch, R. C. E., Verolme, E. K., \& de Zeeuw, P. T. 2006, A\&A, 445, 513

van Loon, J. T., van Leeuwen, F., Smalley, B., et al. 2007, MNRAS, 382, 1353

Weldrake, D. T. F., Sackett, P. D., \& Bridges, T. J. 2007, AJ, 133, 1447 


\section{Appendix A: Catalog}

Table A.1. Catalog of RR Lyrae stars in $\omega$ Cen field, used to derive the empirical PL relations.

\begin{tabular}{|c|c|c|c|c|c|c|c|c|c|c|}
\hline ID & $\begin{array}{l}\text { RA (J2000.0) } \\
\text { hh:mm:ss.ss }\end{array}$ & $\begin{array}{c}\text { Dec (J2000.0) } \\
\text { dd:mm:ss.s }\end{array}$ & $\begin{array}{c}P \\
\text { (days) }\end{array}$ & $\begin{array}{c}J \\
(\mathrm{mag})\end{array}$ & $\begin{array}{c}e \\
(\mathrm{mag})\end{array}$ & $\begin{array}{c}K_{\mathrm{s}} \\
(\mathrm{mag})\end{array}$ & $\begin{array}{c}e \\
(\mathrm{mag})\end{array}$ & Type & $\begin{array}{l}{[\mathrm{Fe} / \mathrm{H}]} \\
(\mathrm{R} 00)\end{array}$ & $\begin{array}{l}{[\mathrm{Fe} / \mathrm{H}]} \\
(\mathrm{S} 06)\end{array}$ \\
\hline V3 & $13: 25: 56.14$ & $-47: 25: 54.2$ & 0.841258 & 13.152 & 0.008 & 12.821 & 0.003 & $\mathrm{RRab}$ & $-1.54 \pm 0.05$ & \\
\hline V4 & $13: 26: 12.91$ & $-47: 24: 19.2$ & 0.627320 & 13.376 & 0.051 & 13.059 & 0.005 & $\mathrm{RRab}$ & $-1.74 \pm 0.05$ & \\
\hline V5 & $13: 26: 18.31$ & $-47: 23: 12.8$ & 0.515274 & 13.626 & 0.032 & 13.362 & 0.005 & $\mathrm{RRab}$ & $-1.35 \pm 0.08$ & $-1.24 \pm 0.11$ \\
\hline V7 & $13: 27: 01.02$ & $-47: 14: 00.1$ & 0.713000 & 13.290 & 0.012 & 12.947 & 0.003 & $\mathrm{RRab}$ & $-1.46 \pm 0.08$ & \\
\hline V8 & $13: 27: 48.43$ & $-47: 28: 20.7$ & 0.521329 & 13.575 & 0.013 & 13.275 & 0.004 & $\mathrm{RRab}$ & $-1.91 \pm 0.28$ & \\
\hline V9 & $13: 25: 59.57$ & $-47: 26: 24.4$ & 0.523315 & 13.694 & 0.025 & 13.399 & 0.006 & RRab & $-1.49 \pm 0.06$ & \\
\hline V10 & $13: 26: 06.99$ & $-47: 24: 37.0$ & 0.375125 & 13.493 & 0.007 & 13.271 & 0.003 & $\mathrm{RRc}$ & $-1.66 \pm 0.10$ & \\
\hline V11 & $13: 26: 30.54$ & $-47: 23: 01.9$ & 0.564798 & 13.415 & 0.008 & 13.168 & 0.004 & $\mathrm{RRab}$ & $-1.67 \pm 0.13$ & $-1.61 \pm 0.22$ \\
\hline V12 & $13: 26: 27.17$ & $-47: 24: 06.6$ & 0.386769 & 13.467 & 0.004 & 13.234 & 0.002 & $\mathrm{RRc}$ & $-1.53 \pm 0.14$ & \\
\hline V14 & $13: 25: 59.66$ & $-47: 39: 09.9$ & 0.377114 & 13.567 & 0.004 & 13.317 & 0.002 & $\mathrm{RRc}$ & $-1.71 \pm 0.13$ & \\
\hline V15 & $13: 26: 27.08$ & $-47: 24: 38.4$ & 0.810642 & 13.133 & 0.013 & 12.824 & 0.002 & $\mathrm{RRab}$ & $-1.64 \pm 0.39$ & $-1.68 \pm 0.18$ \\
\hline V16 & $13: 27: 37.71$ & $-47: 37: 35.2$ & 0.330202 & 13.675 & 0.005 & 13.453 & 0.002 & $\mathrm{RRc}$ & $-1.29 \pm 0.08$ & \\
\hline V18 & $13: 27: 45.06$ & $-47: 24: 56.9$ & 0.621689 & 13.372 & 0.014 & 13.079 & 0.003 & $\mathrm{RRab}$ & $-1.78 \pm 0.28$ & \\
\hline V19 & $13: 27: 30.12$ & $-47: 28: 05.7$ & 0.299551 & 13.857 & 0.004 & 13.632 & 0.002 & $\mathrm{RRc}$ & $-1.22 \pm 0.05$ & \\
\hline V20 & $13: 27: 14.03$ & $-47: 28: 06.9$ & 0.615559 & 13.379 & 0.017 & 13.091 & 0.003 & $\mathrm{RRab}$ & & $-1.52=$ \\
\hline V21 & $13: 26: 11.15$ & $-47: 25: 59.3$ & 0.380812 & 13.535 & 0.011 & 13.358 & 0.003 & $\mathrm{RRc}$ & $-0.90 \pm 0.11$ & \\
\hline V22 & 13: & :08.1 & 27 & 13.508 & & 57 & 0.002 & $\mathrm{RRc}$ & 0.17 & -1.60 \\
\hline $\mathrm{V} 23$ & .48 & 39.6 & 0.510870 & 13.678 & 0.023 & 407 & 0.004 & $\mathrm{RRab}$ & $8 \pm 0.14$ & -1 . \\
\hline V24 & $13: 27: 38.33$ & $-47: 34: 15.0$ & 0.462278 & 13.361 & 0.005 & 13.085 & 0.002 & $\mathrm{RRc}$ & $-1.86 \pm 0.03$ & \\
\hline $\mathrm{V} 25$ & $13: 26: 25.49$ & $-47: 28: 23.7$ & 0.588466 & 13.386 & 0.021 & 13.147 & 0.004 & $\mathrm{RRab}$ & $-1.57 \pm 0.14$ & \\
\hline V26 & $13: 26: 23.61$ & $-47: 26: 59.9$ & 0.784720 & 13.163 & 0.009 & 12.856 & 0.002 & $\mathrm{RRab}$ & $-1.68 \pm 0.10$ & $-1.81 \pm 0.12$ \\
\hline V27 & $13: 26: 26.04$ & $-47: 28: 16.0$ & 0.615680 & 13.499 & 0.012 & 13.193 & 0.003 & $\mathrm{RRab}$ & $-1.50 \pm 0.26$ & \\
\hline V30 & $13: 26: 15.91$ & $-47: 29: 56.5$ & 0.404410 & 13.440 & 0.005 & 13.217 & 0.002 & $\mathrm{RRc}$ & $-1.75 \pm 0.17$ & $-1.62 \pm 0.28$ \\
\hline V32 & $13: 27: 03.34$ & $-47: 21: 39.2$ & 0.620347 & 13.365 & 0.026 & 13.076 & 0.007 & $\mathrm{RRab}$ & $-1.53 \pm 0.16$ & \\
\hline V33 & $13: 25: 51.57$ & $-47: 29: 06.1$ & 0.602324 & 13.426 & 0.015 & 13.122 & 0.004 & $\mathrm{RRab}$ & $-2.09 \pm 0.23$ & -1 \\
\hline V35 & & -47 & & & & & & & 0.08 & \\
\hline V36 & $13: 27: 10.19$ & $-47: 15: 29.5$ & 0.379683 & 13.515 & 0.007 & 13.261 & 0.002 & $\mathrm{RRc}$ & $-1.49 \pm 0.23$ & \\
\hline V38 & $13: 27: 03.23$ & $-47: 36: 30.4$ & 0.779061 & 13.205 & 0.006 & 12.859 & 0.002 & $\mathrm{RRab}$ & $-1.75 \pm 0.18$ & $-1.64 \pm 0.40$ \\
\hline V39 & $13: 27: 59.82$ & $-47: 34: 42.3$ & 0.393374 & 13.533 & 0.004 & 13.271 & 0.002 & RRc & $-1.96 \pm 0.29$ & \\
\hline V40 & $13: 26: 24.54$ & $-47: 30: 46.7$ & 0.634072 & 13.352 & 0.025 & 13.068 & 0.004 & $\mathrm{RRab}$ & $-1.60 \pm 0.08$ & $2 \pm$ \\
\hline V41 & $13: 27: 01.37$ & $-47: 31: 02.0$ & 0.662942 & 13.302 & 0.021 & 13.005 & 0.004 & $\mathrm{RRab}$ & $-1.89 \pm 0.48$ & \\
\hline V44 & $13: 26: 22.38$ & $-47: 34: 35.7$ & 0.567545 & 13.587 & 0.013 & 13.274 & 0.003 & $\mathrm{RRab}$ & $-1.40 \pm 0.12$ & $-1.29 \pm 0.35$ \\
\hline V45 & $13: 25: 30.85$ & $-47: 27: 20.9$ & 0.589116 & 13.441 & 0.016 & 13.134 & 0.004 & $\mathrm{RRab}$ & $-1.78 \pm 0.25$ & \\
\hline V46 & $13: 25: 30.23$ & $-47: 25: 51.7$ & 0.686971 & 13.292 & 0.008 & 12.967 & 0.002 & $\mathrm{RRab}$ & $-1.88 \pm 0.17$ & \\
\hline V47 & $13: 25: 56.47$ & $-47: 24: 12.3$ & 0.485123 & 13.309 & 0.010 & 13.068 & 0.006 & $\mathrm{RRc}$ & $-1.58 \pm 0.31$ & \\
\hline V49 & $13: 26: 07.73$ & $-47: 37: 55.9$ & 0.604627 & 13.475 & 0.013 & 13.138 & 0.003 & $\mathrm{RRab}$ & $-1.98 \pm 0.11$ & \\
\hline V50 & $13: 25: 53.92$ & $-47: 27: 36.2$ & 0.386172 & 13.584 & 0.003 & 13.307 & 0.003 & $\mathrm{RRc}$ & $-1.59 \pm 0.19$ & \\
\hline V51 & $13: 26: 42.58$ & $-47: 24: 21.6$ & 0.574152 & 13.429 & 0.023 & 13.142 & 0.004 & $\mathrm{RRab}$ & $-1.64 \pm 0.21$ & $-1.84 \pm 0.23$ \\
\hline $\mathrm{V} 52^{a}$ & $13: 26: 35.16$ & $-47: 28: 03.8$ & 0.660386 & 12.854 & 0.022 & 12.650 & 0.007 & $\mathrm{RRab}$ & $-1.42 \pm 0.04$ & \\
\hline V54 & $13: 26: 23.50$ & $-47: 18: 48.1$ & 0.772915 & 13.196 & 0.008 & 12.855 & 0.003 & $\mathrm{RRab}$ & $-1.66 \pm 0.12$ & $-1.80 \pm 0.23$ \\
\hline V55 & $13: 25: 45.11$ & $-47: 42: 20.0$ & 0.581724 & 13.582 & 0.014 & 13.266 & 0.004 & $\mathrm{RRab}$ & $-1.23 \pm 0.31$ & \\
\hline V56 & $13: 25: 55.44$ & $-47: 37: 44.4$ & 0.568023 & 13.629 & 0.010 & 13.302 & 0.003 & $\mathrm{RRab}$ & $-1.26 \pm 0.15$ & \\
\hline V57 & $13: 27: 49.42$ & $-47: 36: 50.7$ & 0.794402 & 13.190 & 0.003 & 12.846 & 0.002 & $\mathrm{RRab}$ & $-1.89 \pm 0.14$ & \\
\hline V58 & $13: 26: 13.03$ & $-47: 24: 03.4$ & 0.369880 & & 0.005 & 13.317 & 0.002 & $\mathrm{RRc}$ & $-1.37 \pm 0.18$ & $-1.91 \pm 0.31$ \\
\hline V59 & $13: 26: 18.41$ & $-47: 29: 47.2$ & 0.518506 & 13.622 & 0.010 & 13.363 & 0.006 & $\mathrm{RRab}$ & $-1.00 \pm 0.28$ & \\
\hline V62 & $13: 26: 26.57$ & $-47: 27: 55.9$ & 0.619770 & 13.330 & 0.018 & 13.062 & 0.004 & $\mathrm{RRab}$ & $-1.62 \pm 0.29$ & \\
\hline V63 & $13: 25: 07.87$ & $-47: 36: 53.8$ & 0.825943 & 13.171 & 0.005 & 12.819 & 0.002 & $\mathrm{RRab}$ & $-1.73 \pm 0.09$ & \\
\hline V64 & $13: 26: 02.16$ & $-47: 36: 19.6$ & 0.344458 & 13.627 & 0.005 & 13.382 & 0.002 & $\mathrm{RRc}$ & $-1.46 \pm 0.23$ & \\
\hline V66 & $13: 26: 33.02$ & $-47: 22: 25.5$ & 0.407461 & 13.441 & 0.004 & 13.200 & 0.002 & RRc & $-1.68 \pm 0.34$ & \\
\hline $\mathrm{V} 67^{c}$ & $13: 26: 28.56$ & $-47: 18: 47.2$ & 0.564451 & 13.557 & 0.010 & 13.271 & 0.003 & $\mathrm{RRab}$ & -1.10 & $-1.19 \pm 0.23$ \\
\hline V68 & $13: 26: 12.79$ & $-47: 19: 36.1$ & 0.534696 & 13.175 & 0.005 & 12.896 & 0.002 & $\mathrm{RRc}$ & $-1.60 \pm 0.01$ & \\
\hline V69 & $13: 25: 10.94$ & $-47: 37: 33.2$ & 0.653221 & 13.367 & 0.015 & 13.061 & 0.003 & $\mathrm{RRab}$ & $-1.52 \pm 0.14$ & \\
\hline V70 & $13: 27: 27.75$ & $-47: 33: 43.3$ & 0.390625 & 13.513 & 0.009 & 13.267 & 0.004 & $\mathrm{RRc}$ & $-1.94 \pm 0.15$ & $-1.74 \pm 0.30$ \\
\hline
\end{tabular}

Notes. ${ }^{(a)}$ Stars not considered to derive the PL-Z relations because their mean magnitudes are brighter than the mean locus of RRab or RRc stars (see Fig. 2). These "over-luminous" stars are due to unresolved companions and blending. ${ }^{(b)}$ The $J$ and $K_{\mathrm{S}}$ light curves have a considerable gap in the observations because of the one day period. This star was not considered to derive the PL $-Z$ relations. ${ }^{(c)}$ Stars with metallicity measurements without error reported were not included to derive the PL-Z relations. 
Table A.1. continued.

\begin{tabular}{|c|c|c|c|c|c|c|c|c|c|c|}
\hline ID & $\begin{array}{l}\text { RA (J2000.0) } \\
\text { hh:mm:ss.ss }\end{array}$ & $\begin{array}{c}\text { Dec (J2000.0) } \\
\text { dd:mm:ss.s }\end{array}$ & $\begin{array}{c}P \\
\text { (days) }\end{array}$ & $\begin{array}{c}J \\
(\mathrm{mag})\end{array}$ & $\begin{array}{c}e \\
(\mathrm{mag})\end{array}$ & $\begin{array}{c}K_{\mathrm{s}} \\
(\mathrm{mag})\end{array}$ & $\begin{array}{c}e \\
(\mathrm{mag})\end{array}$ & Type & $\begin{array}{c}{[\mathrm{Fe} / \mathrm{H}]} \\
(\mathrm{R} 00)\end{array}$ & $\begin{array}{l}{[\mathrm{Fe} / \mathrm{H}]} \\
(\mathrm{S} 06) \\
\end{array}$ \\
\hline V71 & $13: 27: 08.05$ & $-47: 27: 52.2$ & 0.357544 & 13.535 & 0.021 & 13.310 & 0.007 & $\mathrm{RRc}$ & & $-1.74 \pm 0.28$ \\
\hline V72 & $13: 27: 33.02$ & $-47: 16: 22.7$ & 0.384522 & 13.524 & 0.004 & 13.278 & 0.002 & $\mathrm{RRc}$ & $-1.32 \pm 0.22$ & \\
\hline V73 & $13: 25: 53.65$ & $-47: 16: 10.7$ & 0.575215 & 13.470 & 0.009 & 13.193 & 0.003 & $\mathrm{RRab}$ & $-1.50 \pm 0.09$ & \\
\hline V74 & $13: 27: 07.25$ & $-47: 17: 34.3$ & 0.503209 & 13.600 & 0.022 & 13.308 & 0.004 & $\mathrm{RRab}$ & $-1.83 \pm 0.36$ & \\
\hline V75 & $13: 27: 19.69$ & $-47: 18: 46.9$ & 0.421980 & 13.390 & 0.005 & 13.125 & 0.002 & $\mathrm{RRc}$ & $-1.49 \pm 0.08$ & $-1.82 \pm 0.99$ \\
\hline V76 & $13: 26: 57.27$ & $-47: 20: 07.9$ & 0.337962 & 13.632 & 0.004 & 13.412 & 0.002 & $\mathrm{RRc}$ & $-1.45 \pm 0.13$ & \\
\hline $\mathrm{V} 77^{c}$ & $13: 27: 20.87$ & $-47: 22: 06.0$ & 0.426136 & 13.423 & 0.004 & 13.146 & 0.002 & $\mathrm{RRc}$ & -1.81 & $-1.84 \pm 0.43$ \\
\hline V79 & $13: 28: 25.08$ & $-47: 29: 24.8$ & 0.608276 & 13.439 & 0.017 & 13.116 & 0.003 & $\mathrm{RRab}$ & $-1.39 \pm 0.18$ & \\
\hline V81 & $13: 27: 36.72$ & $-47: 24: 48.4$ & 0.389392 & 13.507 & 0.004 & 13.255 & 0.002 & $\mathrm{RRc}$ & $-1.72 \pm 0.31$ & $-1.99 \pm 0.43$ \\
\hline V82 & $13: 27: 35.58$ & $-47: 26: 30.8$ & 0.335758 & 13.599 & 0.005 & 13.375 & 0.002 & $\mathrm{RRc}$ & $-1.56 \pm 0.20$ & \\
\hline V83 & $13: 27: 08.42$ & $-47: 21: 34.4$ & 0.356612 & 13.594 & 0.004 & 13.359 & 0.002 & $\mathrm{RRc}$ & $-1.30 \pm 0.22$ & \\
\hline V84 & 47.47 & $-47: 29: 56.2$ & 0.579873 & 13.043 & 0.006 & 12.719 & 0.003 & RRab & $-1.47 \pm 0.10$ & \\
\hline V85 & 06.60 & $-47:$ & 0.742758 & 13.245 & 0.010 & 12.907 & 0.003 & $\mathrm{RRab}$ & $-1.87 \pm 0.31$ & \\
\hline V86 & $13: 27: 15.17$ & $-47: 26: 11.6$ & 0.647844 & 13.357 & 0.015 & 13.043 & 0.003 & $\mathrm{RRab}$ & $-1.81 \pm 0.18$ & 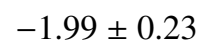 \\
\hline V87 & & -47 & 0.396488 & 13.479 & 0.007 & 13.246 & 0.003 & $\mathrm{RRc}$ & $-1.44 \pm 0.19$ & \\
\hline V88 & $13: 26: 55.89$ & $-47: 25: 16.5$ & 0.690211 & 13.262 & 0.021 & 12.985 & 0.007 & $\mathrm{RRab}$ & $-1.65 \pm 0.23$ & \\
\hline V89 & & & & 13.574 & & & & & $-1.37 \pm 0.28$ & $-1.66 \pm 0.23$ \\
\hline $\mathrm{V} 90^{c}$ & 13 & -4 & 0.603404 & 13.396 & 0.025 & & 0.005 & RRab & -2.21 & .31 \\
\hline V91 & 0.58 & $-47:$ & 0.895225 & 13.010 & 0.008 & 12.686 & 0.004 & $\mathrm{RRab}$ & $-1.44 \pm 0.17$ & \\
\hline V94 & 07 & -47 & 36 & 13.977 & 0.006 & 13.811 & 0.002 & $\mathrm{RRc}$ & $-1.00 \pm 0.11$ & \\
\hline V95 & & -47 & 67 & & 0.005 & & 0.002 & RRc & 0.55 & \\
\hline V96 $6^{c}$ & & & & 13.327 & 0.024 & & 0.004 & RRab & & \\
\hline V97 & 48 & -47 : & 0.691898 & 13.300 & 0.013 & 12.988 & 0.003 & RRab & $-1.56 \pm 0.37$ & \pm 0.17 \\
\hline V98 & & $-47: 26: 57.1$ & 0.280566 & 13.893 & 0.006 & & 0.003 & $\mathrm{RRc}$ & $-1.05 \pm 0.12$ & \\
\hline V99 & & -4 & 81 & 13.110 & 0.024 & 38 & 0.004 & $\mathrm{RRab}$ & 0.14 & $-1.91 \pm 0.25$ \\
\hline V100 & $13: 27: 04.01$ & $-47: 27: 33.8$ & 0.552745 & 13.619 & 0.025 & 13.328 & 0.005 & $\mathrm{RRab}$ & \pm 0.14 & \\
\hline V101 & & & & 13.644 & 0.006 & & 0.002 & & $-1.88 \pm 0.32$ & \\
\hline V102 & 13 & -47 & 0.69 & 13.281 & 0.010 & 77 & 0.003 & RRab & $-1.84 \pm 0.13$ & $-1.65 \pm 0.16$ \\
\hline V103 & 14.26 & $-47: 28: 36.9$ & 0.328852 & 13.600 & 0.004 & & 0.002 & $\mathrm{RRc}$ & $-1.92 \pm 0.11$ & \\
\hline V104 & & -47 & & 13.168 & 0.006 & 24 & 0.003 & $\mathrm{RRab}$ & $-1.83 \pm 0.18$ & \\
\hline V105 & & -47 & & 13.740 & 0.004 & & 0.002 & DO & & \\
\hline V106 & & -47 & 0.569903 & 13.362 & 0.025 & 13 & 0.005 & $\mathrm{RRab}$ & $-1.50 \pm 0.23$ & \\
\hline V107 & 14.02 & $-47: 30: 58.5$ & 0.514102 & 13.670 & 0.016 & 13. & 0.003 & RRab & $-1.36 \pm 0.11$ & \\
\hline V108 & & $-47 \cdot$ & 0.594458 & 13.328 & 0.011 & & 0.004 & $\mathrm{RRab}$ & $=0.23$ & $3 \pm 0.13$ \\
\hline V109 & 13 & $-47: 29: 37.0$ & 0.744098 & 13.193 & 0.020 & 1 & & $\mathrm{RRab}$ & $-1.51 \pm 0.25$ & \\
\hline V110 & & & & & & & & & & \\
\hline V111 & & -47 & & 84 & 0.014 & & 0.003 & RRab & $-1.66 \pm 0.04$ & \pm 0.09 \\
\hline V112 & .24 & $-47: 30: 23.6$ & 0.474359 & 13.574 & 0.027 & 54 & 0.006 & $\mathrm{RRab}$ & $-1.81 \pm 0.26$ & \\
\hline V113 & & 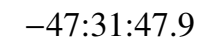 & 75 & 13.447 & 0.025 & & 0005 & PPoh & 0.34 & \\
\hline V114 & 13 & $-47: 30: 21.4$ & 0.675307 & 13.292 & 0.015 & 12.997 & 0.004 & $\mathrm{RRab}$ & $-1.32 \pm 0.30$ & $-1.61 \pm 0.99$ \\
\hline $\mathrm{V}$ & & & & & & & & & & \\
\hline V116 & & $-47:$ & & 13 & $0 .($ & & 0.006 & $\mathrm{RRab}$ & 0.44 & \pm 0.17 \\
\hline V117 & $13: 26$ & $-47: 29: 21.6$ & 0.421641 & 13.439 & 0.005 & 13.191 & 0.003 & $\mathrm{RRc}$ & $-1.68 \pm 0.25$ & \\
\hline & & 17 & 0.305876 & 13.695 & 0.004 & & 0002 & - & $=0.10$ & \\
\hline V120 & 13: & $-47: 3$ & 48537 & 13.560 & 0.0 & 13. & & $\mathrm{RRab}$ & $-1.39 \pm 0.06$ & \\
\hline V1 & & & & & & & & & & \\
\hline V122 & & -47 & & & 0.0 & & $0 .($ & RRab & .18 & $-179+021$ \\
\hline V123 & $13: 26$ & $-47: 37: 13.3$ & 0.473884 & 13.389 & 0.005 & 13.110 & 0.002 & $\mathrm{RRc}$ & $-1.64 \pm 0.01$ & \\
\hline & & & & & & & & & & \\
\hline V125 & $13: 26: 48.98$ & $-47: 41: 03.7$ & 0.592888 & 13.416 & 0.011 & 13.145 & 0.003 & RRab & $-1.67 \pm 0.22$ & $-1.81 \pm 0.38$ \\
\hline V126 & & & & 13.646 & 0.006 & & & $\mathrm{RRc}$ & & \\
\hline V12 & & -47 & & & 0.004 & & 0.002 & $\mathrm{RRc}$ & -1.5 & \\
\hline V128 & $13: 26: 17.72$ & $-47: 30: 13.5$ & 0.834988 & 13.076 & 0.008 & 12.761 & 0.003 & $\mathrm{RRab}$ & $-1.88 \pm 0.04$ & \\
\hline V130 & & & & & & & & RRab & & \\
\hline V131 & $13: 26: 30.04$ & $-47: 29: 41.1$ & 0.392123 & 13.402 & 0.006 & 13.180 & 0.003 & RRc & $-1.56 \pm 0.20$ & $-1.66 \pm 0.48$ \\
\hline V132 & $13: 26: 39.18$ & $-47: 29: 10.0$ & 0.655656 & 13.267 & 0.022 & 12.990 & 0.005 & $\mathrm{RRab}$ & $-1.91 \pm 0.20$ & \\
\hline V1 & 13: & & & 13.339 & 0.0 & & 0.006 & $\mathrm{RRab}$ & $-1.80 \pm 0.41$ & \\
\hline $\mathrm{V}^{135^{a}}$ & $13: 26: 28.06$ & $-47: 29: 18.3$ & 0.632527 & 12.877 & 0.029 & 12.536 & 0.011 & $\mathrm{RRab}$ & -2.20 & \\
\hline V136 & $13: 26: 31.06$ & $-47: 27: 40.9$ & 0.391945 & 13.397 & 0.007 & 13.182 & 0.005 & $\mathrm{RRc}$ & $-1.83 \pm 0.47$ & \pm 0.37 \\
\hline
\end{tabular}


Table A.1. continued.

\begin{tabular}{|c|c|c|c|c|c|c|c|c|c|c|}
\hline ID & $\begin{array}{c}\text { RA (J2000.0) } \\
\text { hh:mm:ss.ss }\end{array}$ & $\begin{array}{c}\text { Dec (J2000.0) } \\
\text { dd:mm:ss.s }\end{array}$ & $\begin{array}{c}P \\
\text { (days) }\end{array}$ & $\begin{array}{c}J \\
(\mathrm{mag})\end{array}$ & $\begin{array}{c}e \\
(\mathrm{mag})\end{array}$ & $\begin{array}{c}K_{\mathrm{s}} \\
(\mathrm{mag})\end{array}$ & $\begin{array}{c}e \\
(\mathrm{mag})\end{array}$ & Type & $\begin{array}{c}{[\mathrm{Fe} / \mathrm{H}]} \\
(\mathrm{R} 00) \\
\end{array}$ & $\begin{array}{l}{[\mathrm{Fe} / \mathrm{H}]} \\
(\mathrm{S} 06) \\
\end{array}$ \\
\hline V137 & $13: 26: 31.52$ & $-47: 27: 04.6$ & 0.334205 & 13.557 & 0.007 & 13.352 & 0.002 & RRc & $-1.19 \pm 0.18$ & \\
\hline $\mathrm{V} 139^{a}$ & $13: 26: 37.75$ & $-47: 27: 35.4$ & 0.676871 & 13.022 & 0.028 & 12.707 & 0.008 & $\mathrm{RRab}$ & $-1.46 \pm 0.04$ & $-1.83 \pm 0.20$ \\
\hline V140 & $13: 26: 42.15$ & $-47: 30: 07.5$ & 0.619849 & 13.355 & 0.019 & 13.087 & 0.006 & $\mathrm{RRab}$ & & $-1.72 \pm 0.15$ \\
\hline V141 & $13: 26: 40.87$ & $-47: 29: 28.2$ & 0.697363 & 13.221 & 0.019 & 12.915 & 0.007 & RRab & $-1.55 \pm 0.36$ & $-2.20 \pm 0.36$ \\
\hline V142 & $13: 26: 42.63$ & $-47: 28: 42.9$ & 0.375877 & 13.520 & 0.014 & 13.284 & 0.006 & $\mathrm{RRc}$ & & $-1.81 \pm 0.24$ \\
\hline V144 & $13: 26: 43.02$ & $-47: 28: 18.0$ & 0.835320 & 13.044 & 0.008 & 12.742 & 0.004 & $\mathrm{RRab}$ & $-1.71 \pm 0.12$ & \\
\hline V145 & $13: 26: 51.21$ & $-47: 31: 08.8$ & 0.373214 & 13.542 & 0.005 & 13.317 & 0.002 & $\mathrm{RRc}$ & $-1.58 \pm 0.07$ & \\
\hline V147 & $13: 27: 15.88$ & $-47: 31: 10.3$ & 0.422615 & 13.387 & 0.006 & 13.166 & 0.002 & $\mathrm{RRc}$ & $-1.66 \pm 0.14$ & \\
\hline V149 & $13: 27: 32.85$ & $-47: 13: 43.3$ & 0.682728 & 13.297 & 0.013 & 12.997 & 0.003 & RRab & $-1.21 \pm 0.24$ & \\
\hline V150 & $13: 27: 40.23$ & $-47: 36: 00.4$ & 0.899302 & 13.086 & 0.008 & 12.766 & 0.003 & RRab & $-1.76 \pm 0.34$ & \\
\hline V151 & $13: 28: 25.31$ & $-47: 16: 00.0$ & 0.407756 & 13.470 & 0.003 & 13.213 & 0.002 & $\mathrm{RRc}$ & $-1.30 \pm 0.24$ & \\
\hline V153 & $13: 26: 49.65$ & $-47: 26: 23.8$ & 0.386245 & 13.511 & 0.006 & 13.281 & 0.003 & $\mathrm{RRc}$ & $-1.38 \pm 0.19$ & \\
\hline V154 & 13:27:03.11 & $-47: 30: 32.4$ & 0.322340 & 13.618 & 0.007 & 13.462 & 0.002 & $\mathrm{RRc}$ & $-1.39 \pm 0.12$ & $-1.49 \pm 0.23$ \\
\hline V155 & $13: 26: 53.64$ & $-47: 24: 42.8$ & 0.413925 & 13.428 & 0.006 & 13.187 & 0.003 & RRc & $-1.46 \pm 0.09$ & \\
\hline V156 & $13: 26: 47.87$ & $-47: 31: 52.6$ & 0.359067 & 13.513 & 0.009 & 13.320 & 0.004 & $\mathrm{RRc}$ & $-1.40 \pm 0.04$ & $-1.51 \pm 0.38$ \\
\hline V157 & $13: 26: 46.45$ & $-47: 27: 17.7$ & 0.406578 & 13.493 & 0.006 & 13.260 & 0.003 & $\mathrm{RRc}$ & $-1.49 \pm 0.10$ & \\
\hline V158 & $13: 26: 45.30$ & $-47: 30: 40.4$ & 0.367276 & 13.543 & 0.009 & 13.336 & 0.003 & RRc & $-1.25 \pm 0.06$ & $-1.64 \pm 0.49$ \\
\hline $\mathrm{V} 160^{c}$ & $13: 25: 36.08$ & $-47: 12: 32.3$ & 0.397527 & 13.497 & 0.004 & 13.237 & 0.002 & RRc & -1.66 & \\
\hline V163 & $13: 25: 49.47$ & $-47: 20: 21.8$ & 0.313196 & 13.701 & 0.005 & 13.493 & 0.002 & RRc & $-1.18 \pm 0.27$ & \\
\hline V169 & $13: 27: 20.45$ & $-47: 23: 59.6$ & 0.319116 & 13.713 & 0.003 & 13.502 & 0.001 & $\mathrm{RRc}$ & & $-1.65 \pm 0.19$ \\
\hline $\mathrm{V} 261^{a}$ & $13: 27: 15.40$ & $-47: 21: 29.9$ & 0.402512 & 13.358 & 0.005 & 13.049 & 0.002 & RRc & & $-1.50 \pm 0.35$ \\
\hline V263 & $13: 26: 13.11$ & $-47: 26: 10.2$ & 1.012158 & 12.970 & 0.004 & 12.625 & 0.002 & $\mathrm{RRab}$ & & $-1.73 \pm 0.19$ \\
\hline V265 & $13: 26: 30.19$ & $-47: 28: 45.6$ & 0.422600 & 13.384 & 0.02 & 13.129 & 0.004 & $\mathrm{RRc}$ & & $-2.00 \pm 0.29$ \\
\hline V267 & $13: 26: 40.18$ & $-47: 26: 36.0$ & 0.315822 & 13.624 & 0.008 & 13.456 & 0.002 & $\mathrm{RRc}$ & & $-1.62 \pm 0.63$ \\
\hline V268 & $13: 26: 35.11$ & $-47: 26: 11.2$ & 0.8129220 & 13.145 & 0.006 & 12.822 & 0.002 & $\mathrm{RRab}$ & & $-1.76 \pm 0.24$ \\
\hline V271 & $13: 26: 47.10$ & $-47: 30: 04.3$ & 0.4432000 & 13.337 & 0.008 & 13.100 & 0.003 & $\mathrm{RRc}$ & & $-1.80 \pm 0.21$ \\
\hline V275 & $13: 26: 49.72$ & $-47: 27: 37.4$ & 0.3778970 & 13.526 & 0.009 & 13.314 & 0.003 & RRc & & $-1.66 \pm 0.36$ \\
\hline V341 & $13: 26: 54.63$ & $-47: 28: 48.4$ & 0.3061360 & 13.621 & 0.017 & 13.366 & 0.015 & RRc & & $-1.78 \pm 0.59$ \\
\hline V342 & $13: 27: 18.72$ & $-47: 28: 22.6$ & 0.3083890 & 13.687 & 0.005 & 13.485 & 0.002 & RRc & & $-1.71 \pm 0.55$ \\
\hline V346 & $13: 26: 46.91$ & $-47: 28: 14.3$ & 0.3276230 & 13.581 & 0.012 & 13.402 & 0.004 & RRc & & $-1.52 \pm 0.54$ \\
\hline V347 & $13: 26: 50.82$ & $-47: 27: 46.2$ & 0.3288490 & 13.637 & 0.025 & 13.459 & 0.009 & RRc & & $-1.66 \pm 0.27$ \\
\hline V350 & $13: 26: 56.44$ & $-47: 30: 50.2$ & 0.3791080 & 13.442 & 0.012 & 13.236 & 0.005 & $\mathrm{RRc}$ & & $-1.45 \pm 0.40$ \\
\hline $\mathrm{V} 353^{a}$ & $13: 26: 43.61$ & $-47: 27: 57.6$ & 0.4010200 & 13.245 & 0.026 & 12.977 & 0.014 & $\mathrm{RRc}$ & & $-1.93 \pm 0.31$ \\
\hline V354 & $13: 26: 38.58$ & $-47: 25: 10.2$ & 0.4200900 & 13.419 & 0.009 & 13.182 & 0.004 & RRc & & $-1.73 \pm 0.23$ \\
\hline V357 & $13: 26: 17.73$ & $-47: 30: 23.0$ & 0.2977750 & 13.690 & 0.005 & 13.510 & 0.003 & RRc & & $-1.64 \pm 0.99$ \\
\hline $\mathrm{V} 366^{b}$ & $13: 26: 41.54$ & $-47: 31: 42.2$ & 0.9999100 & 12.748 & 0.007 & 12.423 & 0.004 & $\mathrm{RRab}$ & & $-1.61 \pm 0.14$ \\
\hline V399 & $13: 26: 29.51$ & $-47: 30: 03.0$ & 0.3097820 & 13.659 & 0.007 & 13.495 & 0.003 & $\mathrm{RRc}$ & & $-1.70 \pm 0.67$ \\
\hline
\end{tabular}


Table A.2. Catalog of fundamental-mode candidate SX Phe stars in $\omega$ Cen, used to derive the empirical PL relations.

\begin{tabular}{|c|c|c|c|c|c|c|c|c|}
\hline ID & $\begin{array}{l}\text { RA (J2000.0) } \\
\text { hh:mm:ss.ss }\end{array}$ & $\begin{array}{c}\text { Dec (J2000.0) } \\
\text { dd:mm:ss.s }\end{array}$ & $\begin{array}{c}P \\
\text { (days) }\end{array}$ & $\begin{array}{c}J \\
(\mathrm{mag})\end{array}$ & $\begin{array}{c}e \\
(\mathrm{mag})\end{array}$ & $\begin{array}{c}K_{\mathrm{s}} \\
(\mathrm{mag})\end{array}$ & $\begin{array}{c}e \\
(\mathrm{mag})\end{array}$ & Remarks $^{a}$ \\
\hline V194 & $13: 27: 53.95$ & $-47: 31: 54.2$ & 0.0471777 & 16.237 & 0.007 & 16.106 & 0.006 & 1 \\
\hline V195 & $13: 27: 15.63$ & $-47: 24: 34.9$ & 0.0654912 & 15.808 & 0.006 & 15.591 & 0.005 & 1 \\
\hline V196 & $13: 25: 01.21$ & $-47: 25: 29.6$ & 0.0574000 & 16.080 & 0.009 & 15.858 & 0.010 & 1 \\
\hline V197 & $13: 26: 20.40$ & $-47: 31: 59.7$ & 0.0471210 & 16.153 & 0.044 & 15.980 & 0.067 & 2 \\
\hline V198 & $13: 26: 34.53$ & $-47: 31: 03.7$ & 0.0481817 & 16.348 & 0.044 & 16.212 & 0.058 & 2 \\
\hline V199 & $13: 26: 28.63$ & $-47: 28: 38.4$ & 0.0622866 & 15.669 & 0.007 & 15.468 & 0.004 & 1 \\
\hline V201 & $13: 26: 11.09$ & $-47: 15: 54.1$ & 0.0506500 & 16.250 & 0.029 & 16.061 & 0.054 & 2 \\
\hline V202 & $13: 26: 38.97$ & $-47: 11: 51.2$ & 0.0464200 & 16.319 & 0.018 & 16.125 & 0.037 & 2 \\
\hline V204 & $13: 27: 07.90$ & $-47: 37: 05.4$ & 0.0493757 & 16.110 & 0.008 & 15.960 & 0.008 & 1 \\
\hline V217 & $13: 26: 16.91$ & $-47: 27: 25.9$ & 0.0532600 & 16.076 & 0.029 & 15.859 & 0.037 & 2 \\
\hline V218 & $13: 26: 11.22$ & $-47: 17: 54.1$ & 0.0437393 & 16.335 & 0.019 & 16.177 & 0.035 & 2 \\
\hline V219 & $13: 26: 08.43$ & $-47: 19: 24.6$ & 0.0386681 & 16.544 & 0.022 & 16.455 & 0.048 & 2 \\
\hline V220 & $13: 26: 48.61$ & $-47: 21: 42.4$ & 0.0528868 & 16.127 & 0.007 & 15.936 & 0.006 & 1 \\
\hline V222 & $13: 26: 18.52$ & $-47: 41: 12.5$ & 0.0389100 & 16.475 & 0.022 & 16.350 & 0.039 & 2 \\
\hline V225 & $13: 27: 02.28$ & $-47: 24: 36.8$ & 0.0486381 & 16.055 & 0.011 & 15.918 & 0.008 & 1 \\
\hline V226 & $13: 26: 10.47$ & $-47: 29: 57.3$ & 0.0378524 & 16.529 & 0.027 & 16.422 & 0.049 & 2 \\
\hline V227 & $13: 26: 14.36$ & $-47: 23: 54.2$ & 0.0382255 & 16.508 & 0.027 & 16.346 & 0.047 & 2 \\
\hline V228 & $13: 26: 40.48$ & $-47: 33: 46.6$ & 0.0398531 & 16.456 & 0.027 & 16.347 & 0.056 & 2 \\
\hline V229 & $13: 27: 12.60$ & $-47: 23: 58.9$ & 0.0375333 & 16.524 & 0.023 & 16.351 & 0.039 & 2 \\
\hline V231 & $13: 27: 19.03$ & $-47: 35: 54.4$ & 0.0374850 & 16.601 & 0.024 & 16.479 & 0.048 & 2 \\
\hline V232 & $13: 26: 17.32$ & $-47: 11: 49.9$ & 0.0369700 & 16.647 & 0.022 & 16.506 & 0.042 & 2 \\
\hline V233 & $13: 26: 47.28$ & $-47: 20: 32.0$ & 0.0365377 & 16.478 & 0.027 & 16.386 & 0.056 & 2 \\
\hline V237 & $13: 25: 52.88$ & $-47: 23: 56.0$ & 0.0656024 & 15.905 & 0.007 & 15.675 & 0.004 & 1 \\
\hline V238 & $13: 27: 33.96$ & $-47: 29: 23.4$ & 0.0408004 & 16.484 & 0.021 & 16.372 & 0.044 & 2 \\
\hline V249 & $13: 25: 46.27$ & $-47: 26: 28.8$ & 0.0349468 & 16.624 & 0.025 & 16.425 & 0.041 & 2 \\
\hline V250 & $13: 27: 31.74$ & $-47: 35: 42.1$ & 0.0406269 & 16.548 & 0.024 & 16.388 & 0.045 & 2 \\
\hline V252 & $13: 27: 29.71$ & $-47: 29: 00.5$ & 0.0466226 & 16.398 & 0.019 & 16.178 & 0.031 & 2 \\
\hline V253 & $13: 27: 22.13$ & $-47: 27: 52.3$ & 0.0399687 & 16.421 & 0.023 & 16.285 & 0.044 & 2 \\
\hline V260 & $13: 24: 52.73$ & $-47: 25: 22.0$ & 0.0462600 & 16.271 & 0.018 & 16.073 & 0.028 & 2 \\
\hline V299 & 13:27:00.30 & $-47: 22: 29.9$ & 0.0344409 & 16.654 & 0.034 & 16.477 & 0.063 & 2 \\
\hline V300 & $13: 25: 46.55$ & $-47: 24: 00.7$ & 0.0347301 & 16.640 & 0.023 & 16.437 & 0.040 & 2 \\
\hline V304 & $13: 26: 19.57$ & $-47: 20: 01.6$ & 0.0361405 & 16.488 & 0.028 & 16.314 & 0.045 & 2 \\
\hline V305 & $13: 27: 51.42$ & $-47: 19: 51.4$ & 0.0365673 & 16.474 & 0.022 & 16.266 & 0.035 & 2 \\
\hline V306 & $13: 27: 10.99$ & $-47: 28: 24.8$ & 0.0384044 & 16.551 & 0.033 & 16.364 & 0.069 & 2 \\
\hline V308 & $13: 27: 50.03$ & $-47: 21: 16.1$ & 0.0389852 & 16.480 & 0.022 & 16.324 & 0.037 & 2 \\
\hline V311 & $13: 26: 58.75$ & $-47: 29: 49.9$ & 0.0414133 & 16.295 & 0.078 & 15.976 & 0.118 & 2 \\
\hline V313 & $13: 26: 38.28$ & $-47: 31: 37.3$ & 0.0418484 & 16.443 & 0.034 & 16.277 & 0.065 & 2 \\
\hline V316 & $13: 26: 14.84$ & $-47: 31: 10.8$ & 0.0424040 & 16.405 & 0.025 & 16.264 & 0.041 & 2 \\
\hline V319 & $13: 26: 28.52$ & $-47: 31: 02.9$ & 0.0489421 & 16.304 & 0.028 & 16.139 & 0.056 & 2 \\
\hline V320 & $13: 26: 49.43$ & $-47: 34: 29.1$ & 0.0471934 & 16.351 & 0.028 & 16.181 & 0.038 & 2 \\
\hline V322 & $13: 26: 38.85$ & $-47: 27: 39.1$ & 0.0479562 & 16.009 & 0.037 & 15.853 & 0.052 & 2 \\
\hline V326 & $13: 26: 40.17$ & $-47: 24: 55.7$ & 0.0569058 & 15.994 & 0.026 & 15.817 & 0.035 & 2 \\
\hline V419/W22 & $13: 28: 53.57$ & $-47: 19: 29.7$ & 0.0410000 & 16.403 & 0.020 & 16.236 & 0.032 & 2 \\
\hline V420/W23 & $13: 28: 44.57$ & $-47: 24: 51.0$ & 0.0370000 & 16.472 & 0.021 & 16.322 & 0.036 & 2 \\
\hline V445/W131 & $13: 24: 54.09$ & $-47: 41: 03.5$ & 0.0470000 & 16.350 & 0.020 & 16.146 & 0.030 & 2 \\
\hline
\end{tabular}

Notes. ${ }^{(a)} 1$ : Variability recovered in our data; 2 : variability not recovered in our data. 